\title{
Low-Coordinate Iron Chalcogenolates and Their Complexes with Diethyl Ether and Ammonia
}

Cary R. Stennett, James C. Fettinger, and Philip P. Power*

Department of Chemistry, University of California, Davis, One Shields Avenue, Davis, California 95616, United States

\section{Supporting Information}

The Supporting Information contains:

1. ${ }^{1} \mathrm{H}-\mathrm{NMR}$ spectra S3-S6

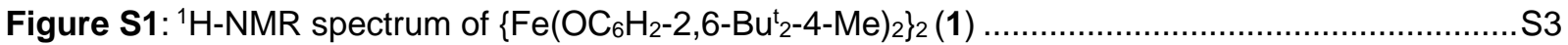

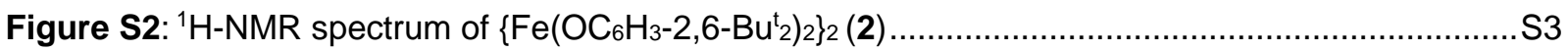

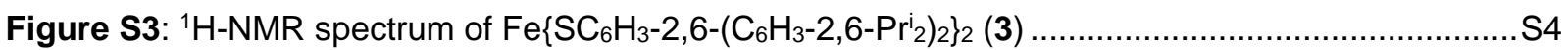

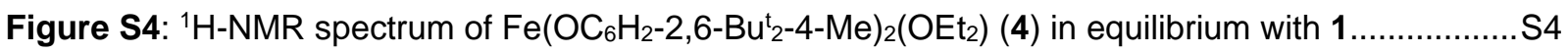

Figure S5: ${ }^{1} \mathrm{H}-\mathrm{NMR}$ spectrum of $\mathrm{Fe}\left(\mathrm{OC}_{6} \mathrm{H}_{3}-2,6-\mathrm{Bu}_{2}\right)_{2}\left(\mathrm{OEt}_{2}\right)(5)$ in equilibrium with (2).......................S5

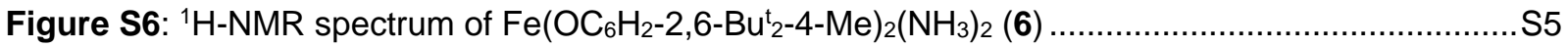

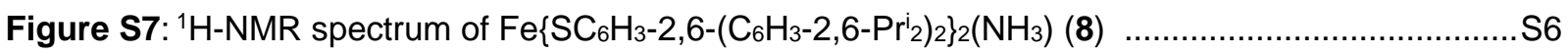

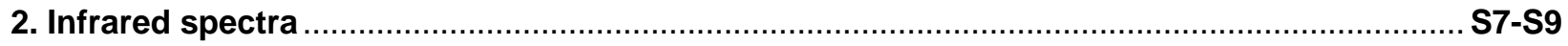

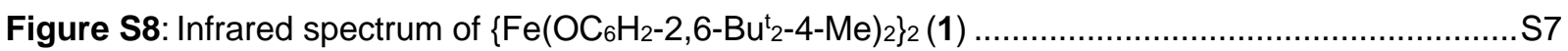

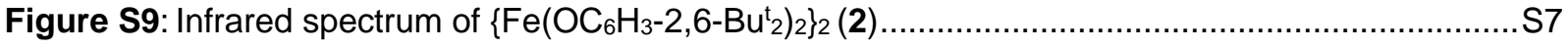

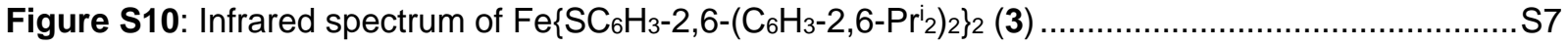

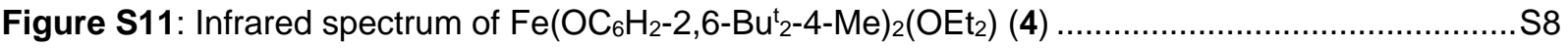

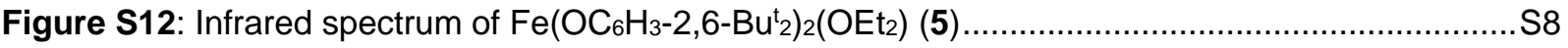

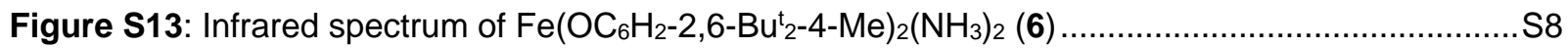

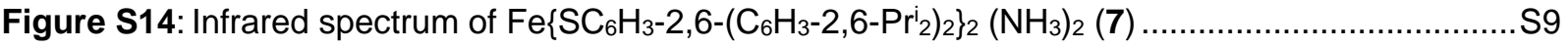

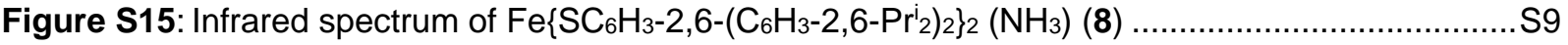

3. Electronic spectra S10-S12

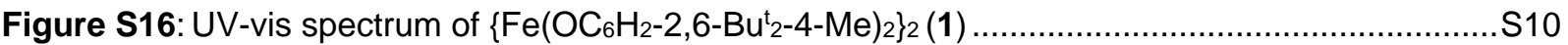

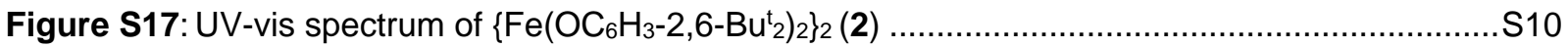

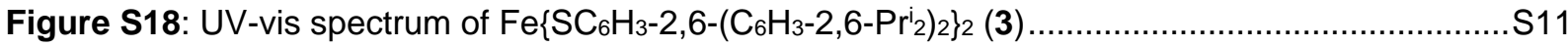




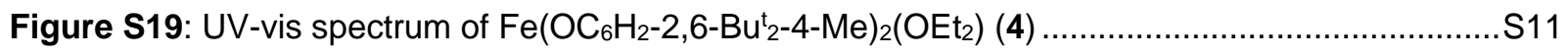

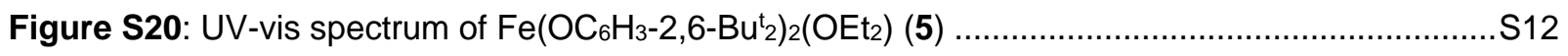

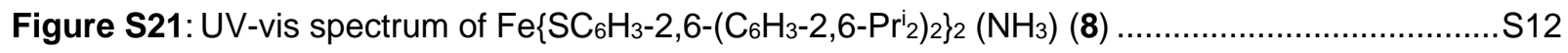

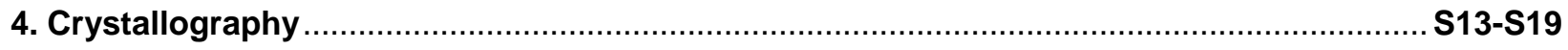

Table S1: Crystallographic and X-ray Data Collection Parameters of Complex 1 ............................. 13

Table S2: Crystallographic and X-ray Data Collection Parameters of Complex 2 ............................S14

Table S3: Crystallographic and X-ray Data Collection Parameters of Complex 4 ............................ 15

Table S4: Crystallographic and X-ray Data Collection Parameters of Complex 5 ............................ 16

Table S5: Crystallographic and X-ray Data Collection Parameters of Complex 6 ............................ 17

Table S6: Crystallographic and X-ray Data Collection Parameters of Complex 7 ............................ 18

Table S7: Crystallographic and X-ray Data Collection Parameters of Complex 8 ...........................S19

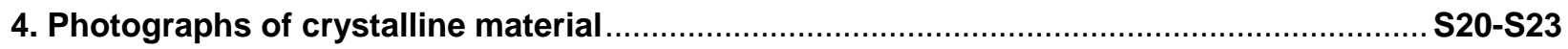

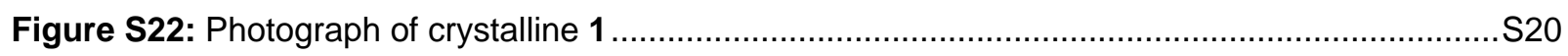

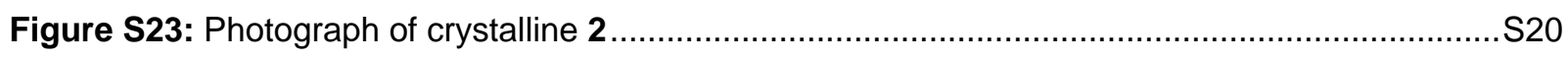

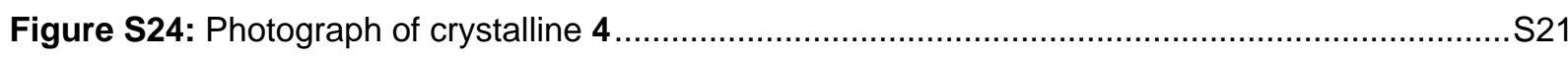

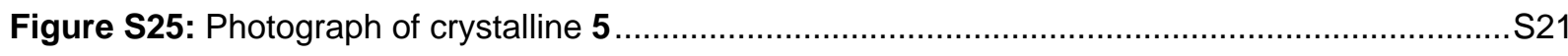

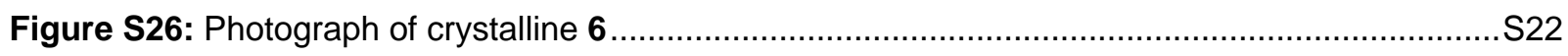

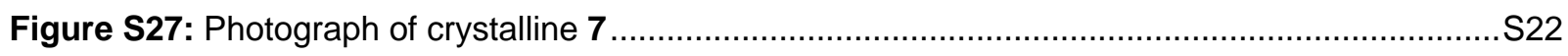

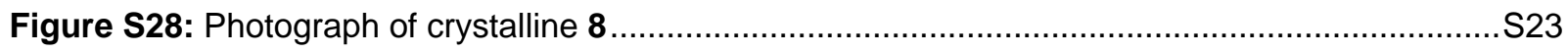




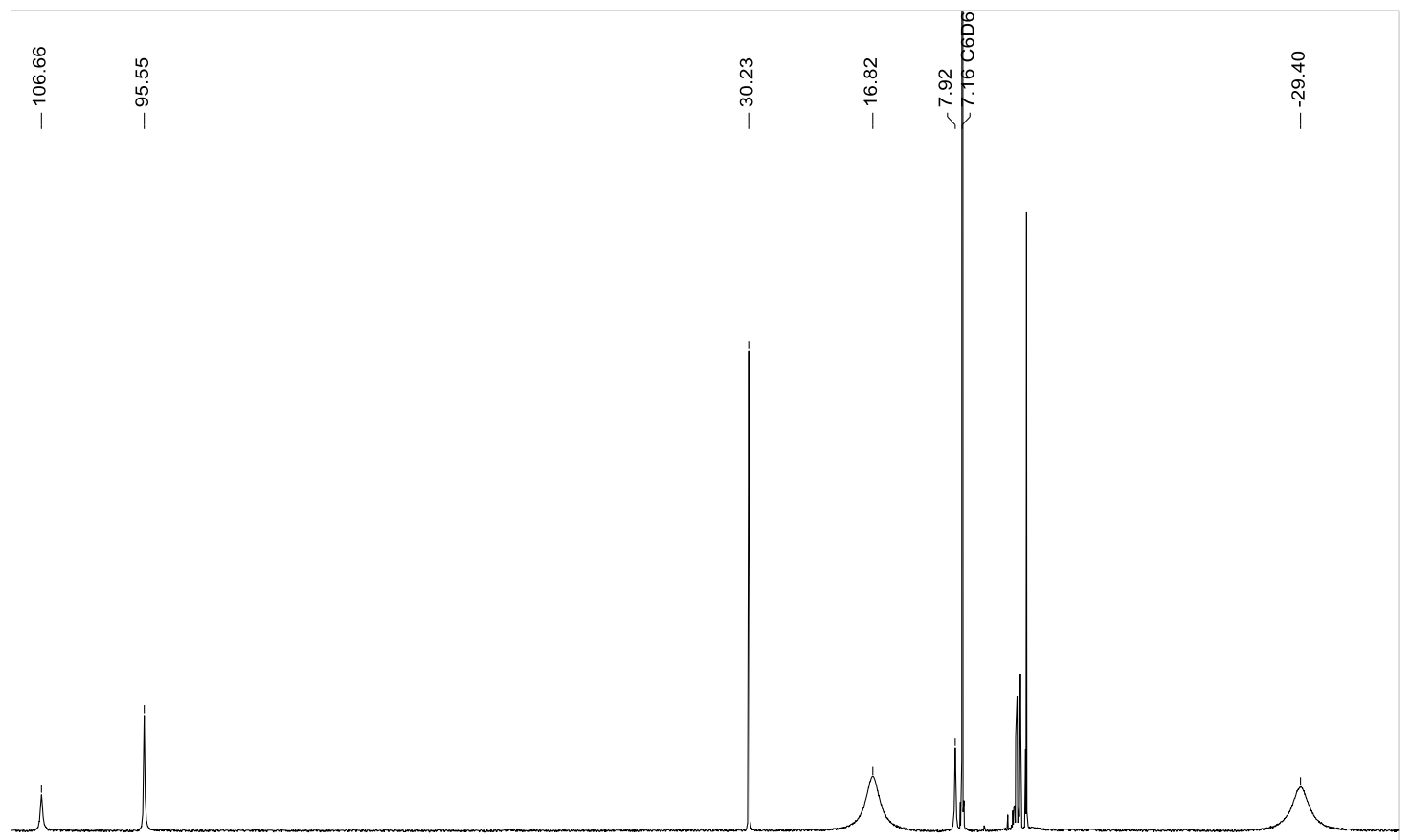

$\begin{array}{llllllllllllllllllllllllllllllllllll}105 & 100 & 95 & 90 & 85 & 80 & 75 & 70 & 65 & 60 & 55 & 50 & 45 & 40 & 35 & 30 & 25 & 20 & 15 & 10 & 5 & 0 & -5 & -10 & -15 & -20 & -25 & -30 & -35\end{array}$ f1 $(\mathrm{ppm})$

Figure S1: ${ }^{1} \mathrm{H}-\mathrm{NMR}$ spectrum of $\left\{\mathrm{Fe}\left(\mathrm{OC}_{6} \mathrm{H}_{2}-2,6-\mathrm{Bu}^{\mathrm{t}}{ }_{2}-4-\mathrm{Me}\right)_{2}\right\}_{2}(1)$ in $\left[\mathrm{D}_{6}\right]$ benzene at $25^{\circ} \mathrm{C}$

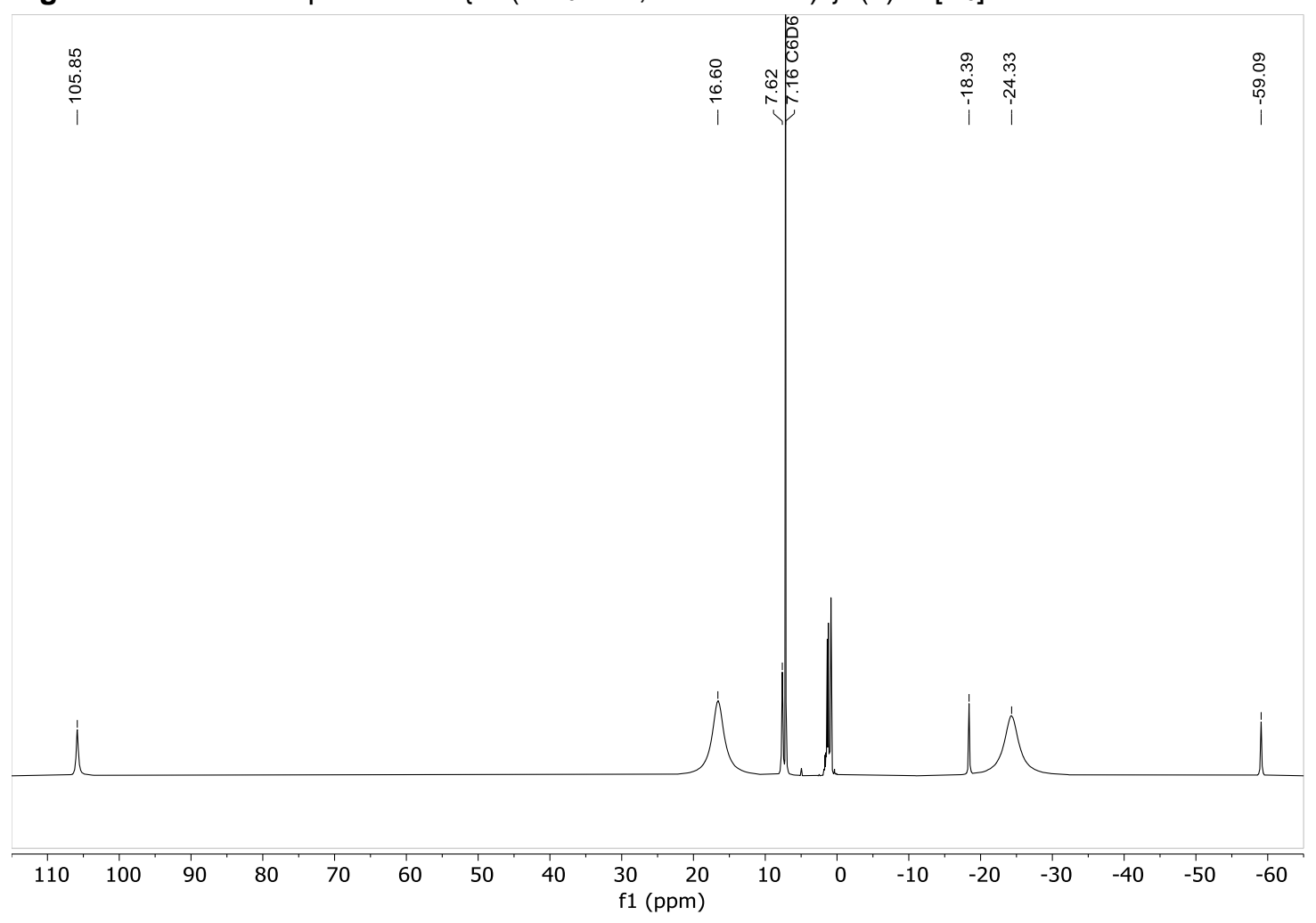

Figure S2: ${ }^{1} \mathrm{H}-\mathrm{NMR}$ spectrum of $\left\{\mathrm{Fe}\left(\mathrm{OC}_{6} \mathrm{H}_{3}-2,6-\mathrm{Bu}_{2}{ }_{2}\right)_{2}\right\}_{2}(2)$ in $\left[\mathrm{D}_{6}\right]$ benzene at $25^{\circ} \mathrm{C}$ 


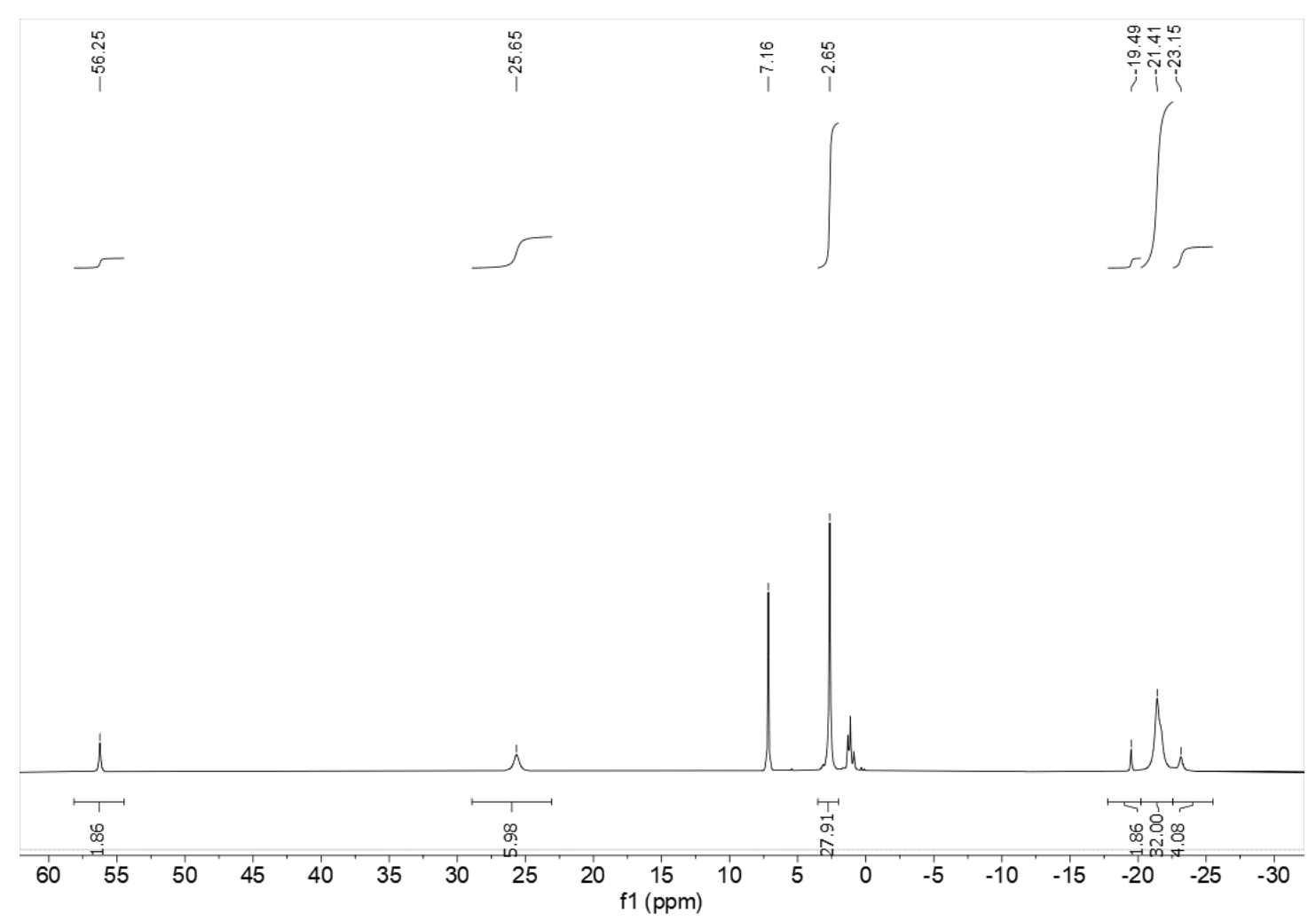

Figure S3: ${ }^{1} \mathrm{H}-\mathrm{NMR}$ spectrum of $\mathrm{Fe}\left\{\mathrm{SC}_{6} \mathrm{H}_{3}-2,6-\left(\mathrm{C}_{6} \mathrm{H}_{3}-2,6-\mathrm{Pr}_{2}\right)_{2}\right\}_{2}(3)$ in $\left[\mathrm{D}_{6}\right]$ benzene at $25^{\circ} \mathrm{C}$

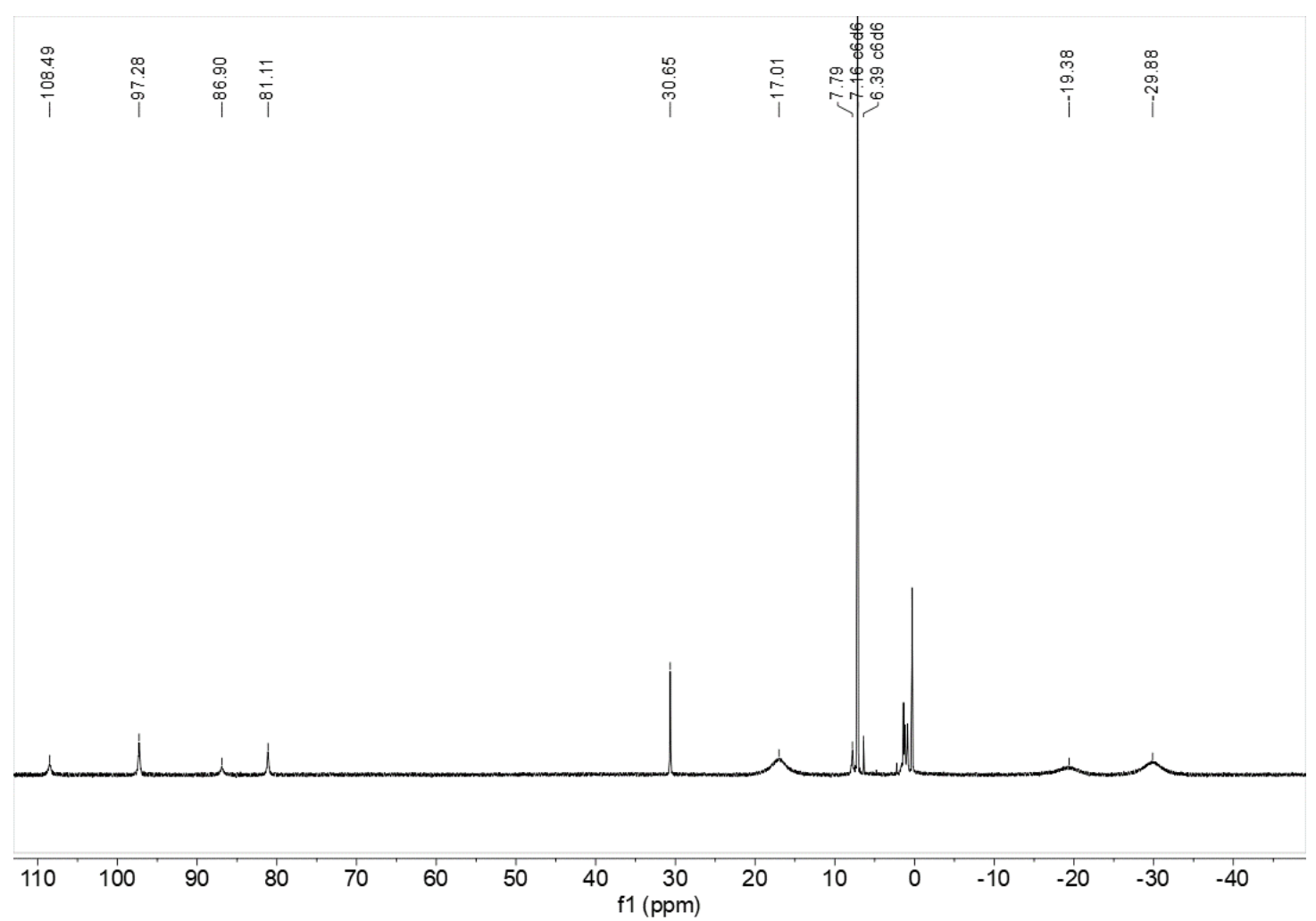

Figure S4: ${ }^{1} \mathrm{H}-\mathrm{NMR}$ spectrum of $\mathrm{Fe}\left(\mathrm{OC}_{6} \mathrm{H}_{2}-2,6-\mathrm{Bu}_{2}{ }_{2}-4-\mathrm{Me}\right)_{2}\left(\mathrm{OEt}_{2}\right)(4)$ in equilibrium with 1 in [D6] benzene at $25^{\circ} \mathrm{C}$. Signal at 6.39 is due to deuterated benzene used for Evans' method. 


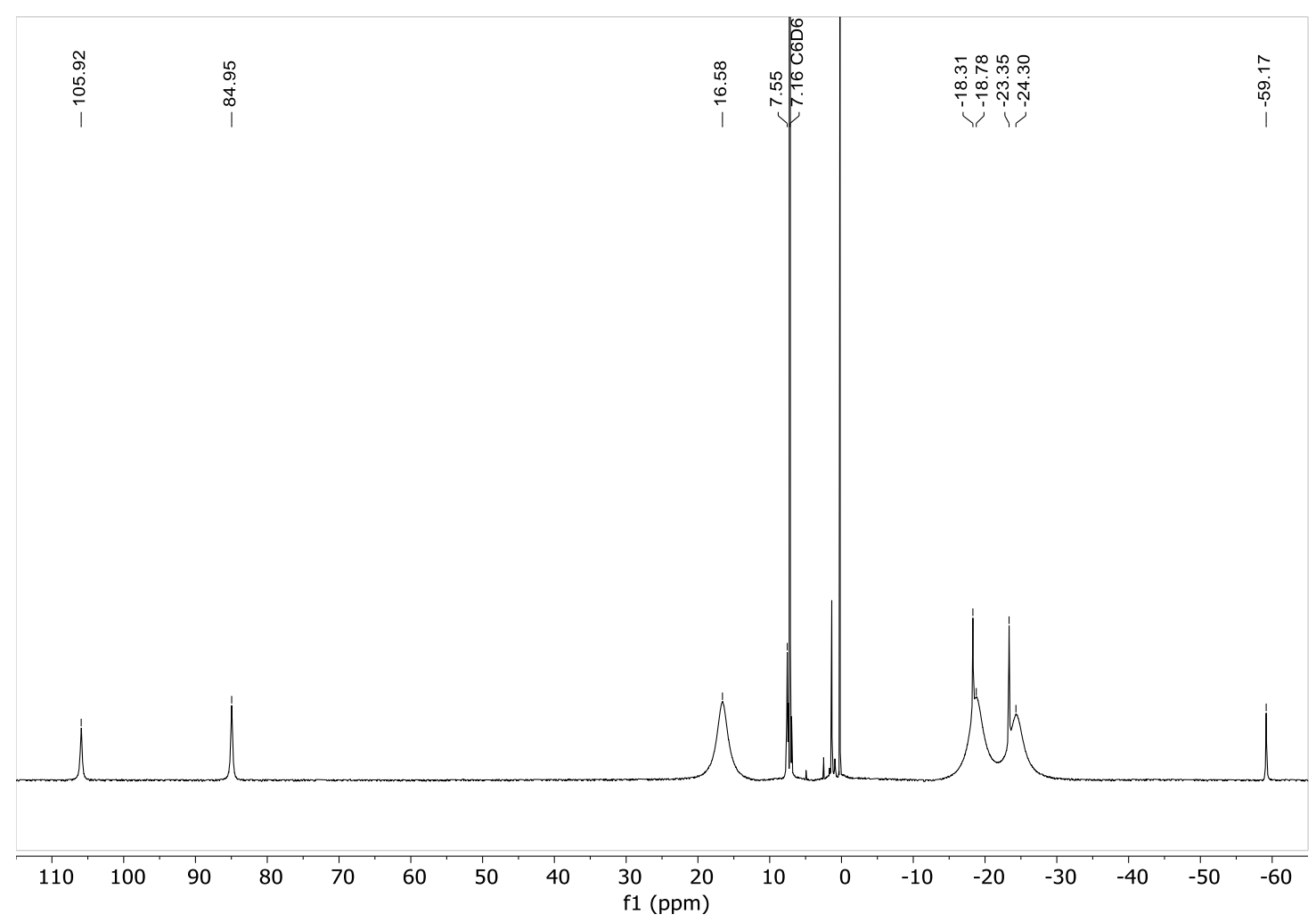

Figure S5: ${ }^{1} \mathrm{H}-\mathrm{NMR}$ spectrum of $\mathrm{Fe}\left(\mathrm{OC}_{6} \mathrm{H}_{3}-2,6-\mathrm{Bu}_{2}\right)_{2}\left(\mathrm{OEt}_{2}\right)(5)$ in equilibrium with (2) in $\left[\mathrm{D}_{6}\right]$ benzene at $25^{\circ} \mathrm{C}$

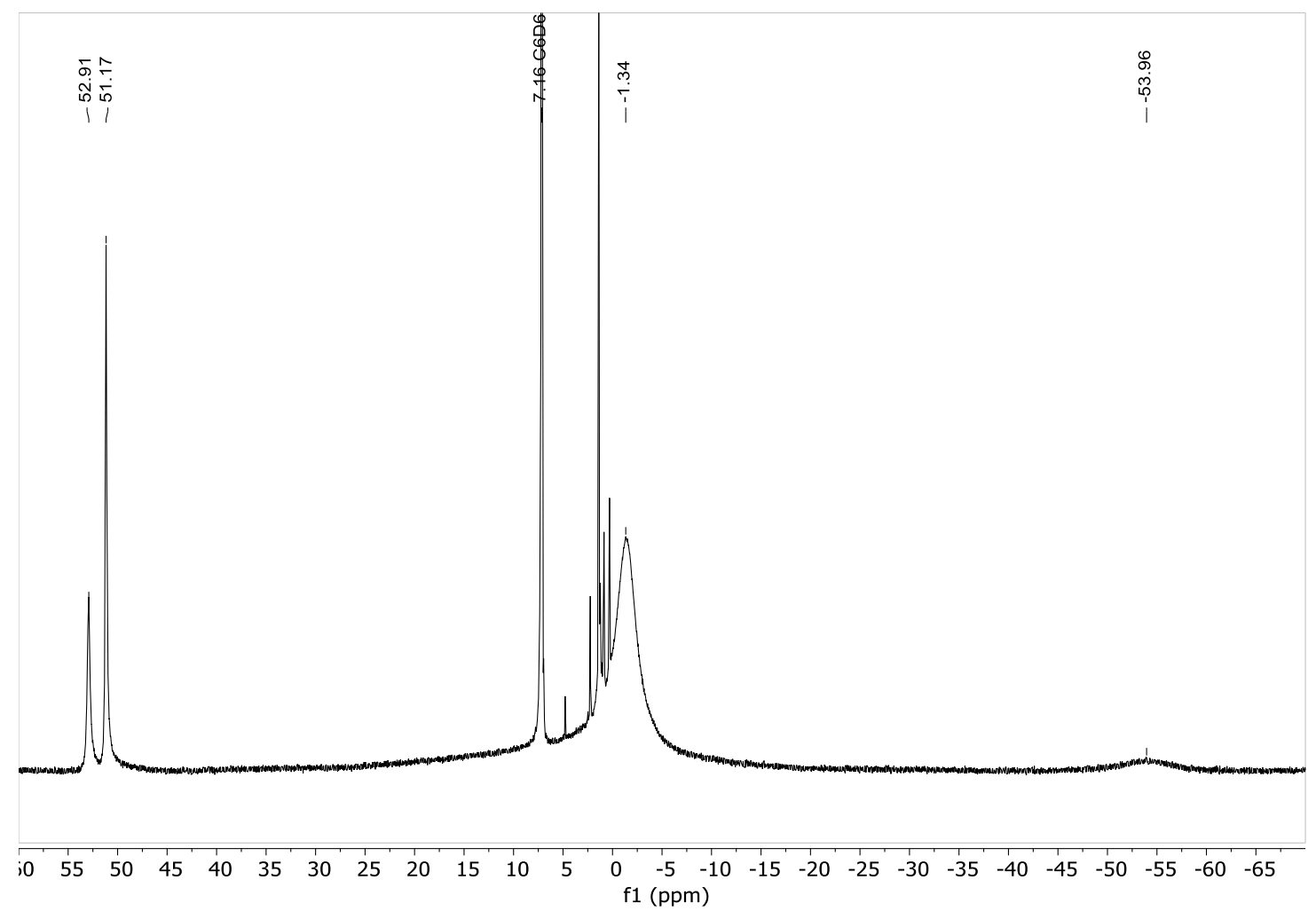

Figure S6: ${ }^{1} \mathrm{H}-\mathrm{NMR}$ spectrum of $\mathrm{Fe}\left(\mathrm{OC}_{6} \mathrm{H}_{2}-2,6-\mathrm{Bu}_{2}{ }_{2}-4-\mathrm{Me}\right)_{2}\left(\mathrm{NH}_{3}\right)_{2}(6)$ in $\left[\mathrm{D}_{6}\right]$ benzene at $25{ }^{\circ} \mathrm{C}$ 


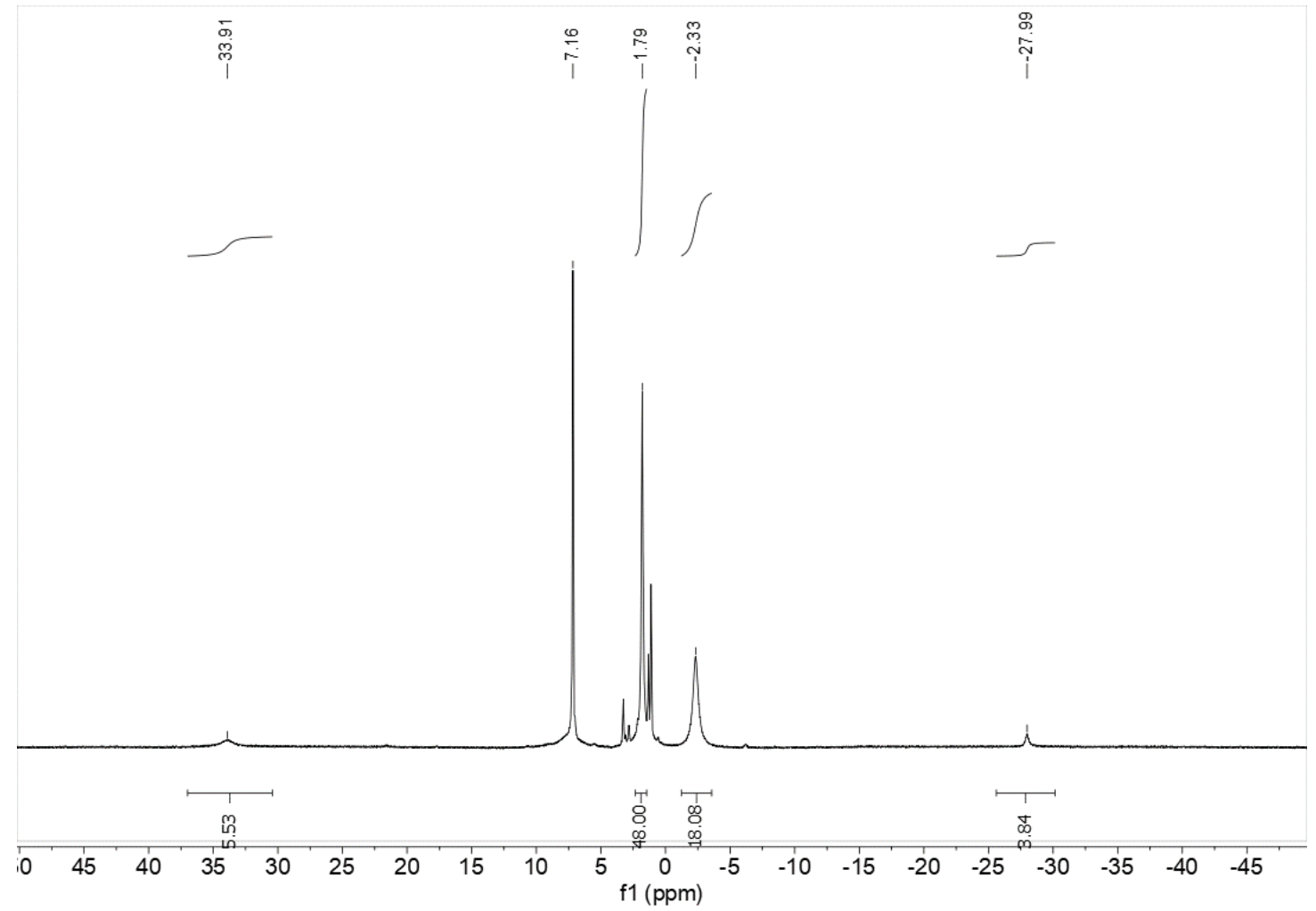

Figure S7: ${ }^{1} \mathrm{H}-\mathrm{NMR}$ spectrum of $\mathrm{Fe}\left\{\mathrm{SC}_{6} \mathrm{H}_{3}-2,6-\left(\mathrm{C}_{6} \mathrm{H}_{3}-2,6-\mathrm{Pr}_{2}\right)_{2}\right\}_{2}\left(\mathrm{NH}_{3}\right)(\mathbf{8})$ in $\left[\mathrm{D}_{6}\right]$ benzene at $25^{\circ} \mathrm{C}$ 


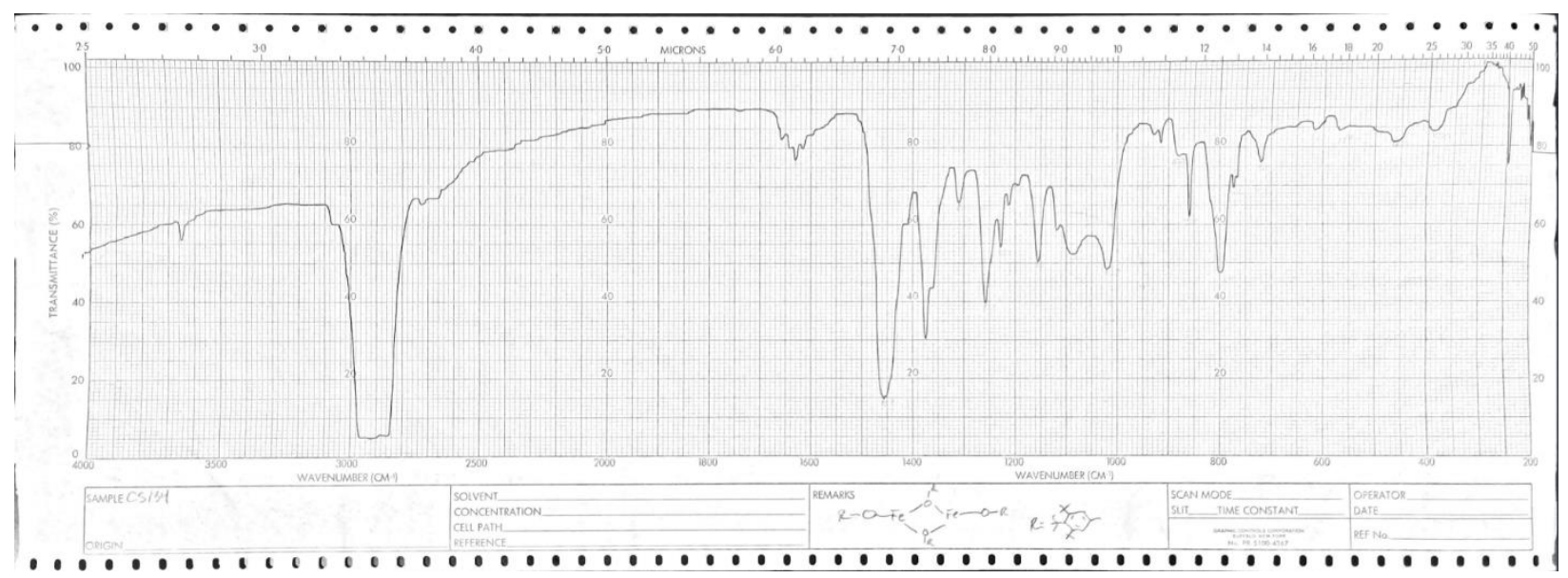

Figure S8: Infrared spectrum of $\left\{\mathrm{Fe}\left(\mathrm{OC}_{6} \mathrm{H}_{2}-2,6-\mathrm{Bu}^{\mathrm{t}} 2-4-\mathrm{Me}\right)_{2}\right\}_{2}$ (1) (Nujol mull, CsI windows)

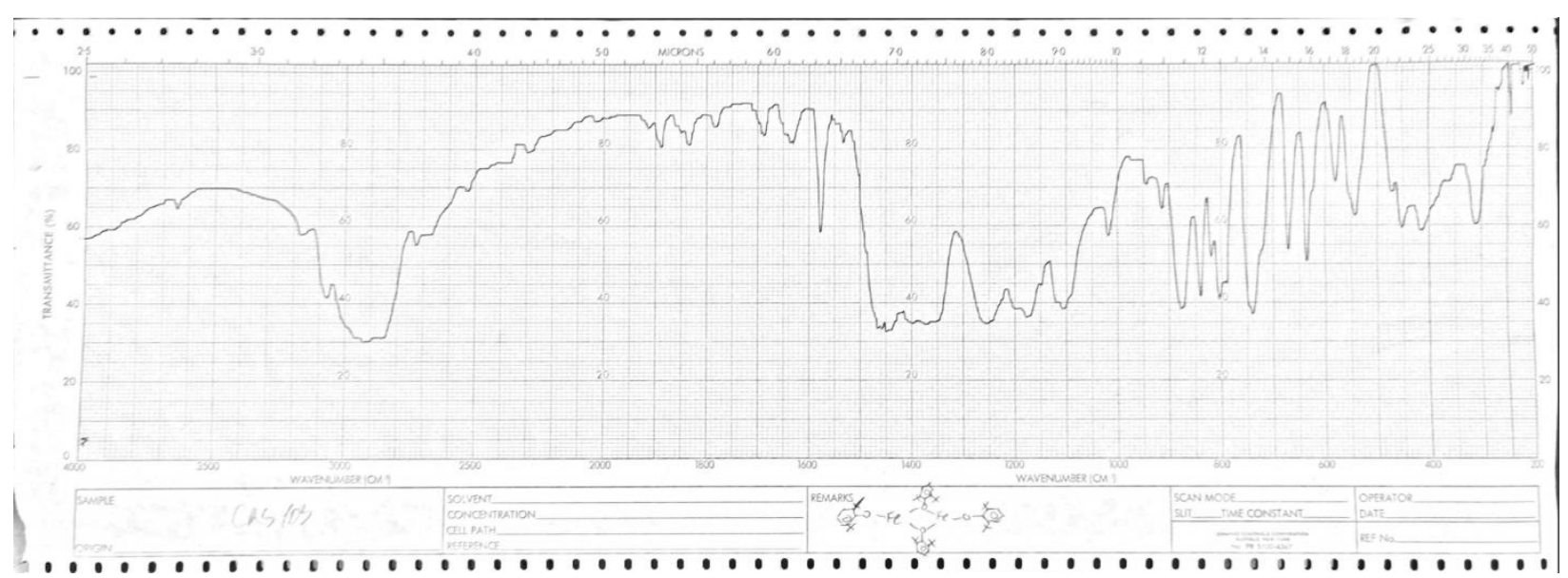

Figure S9: Infrared spectrum of $\left\{\mathrm{Fe}\left(\mathrm{OC}_{6} \mathrm{H}_{3}-2,6-\mathrm{Bu}_{2}^{\mathrm{t}}\right)_{2}\right\}_{2}$ (2) (Nujol mull, CsI windows)

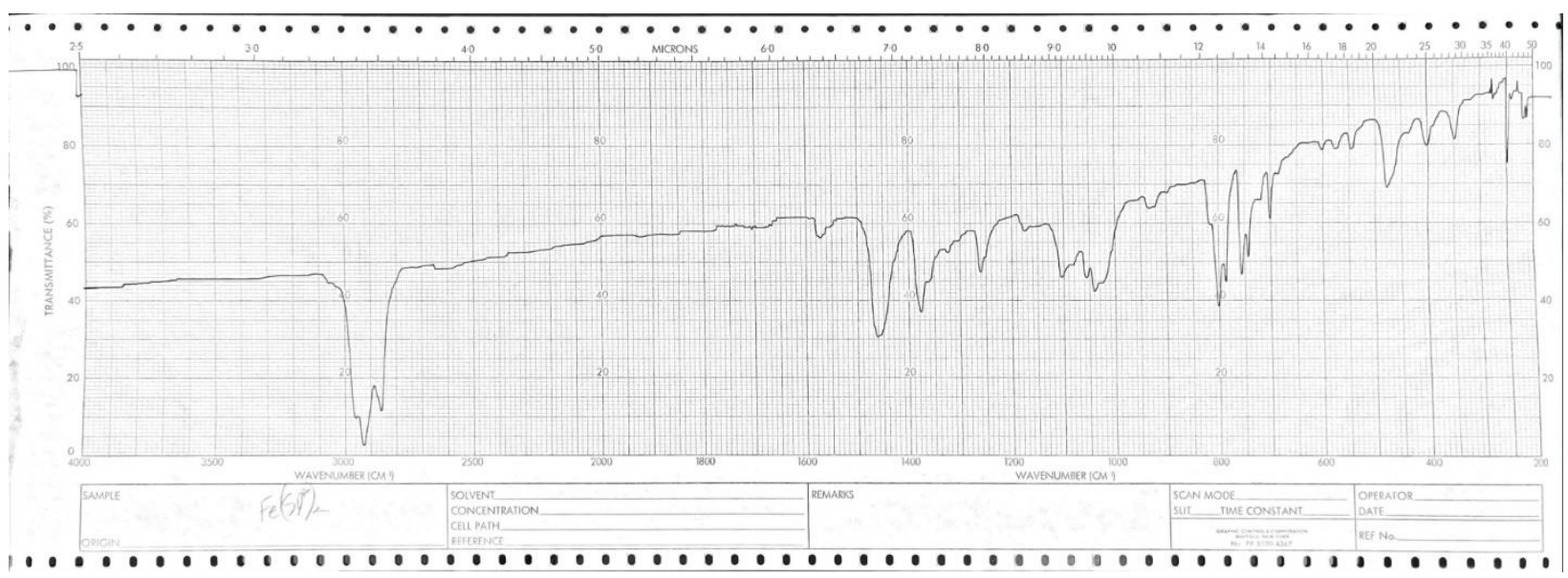

Figure S10: Infrared spectrum of $\mathrm{Fe}\left\{\mathrm{SC}_{6} \mathrm{H}_{3}-2,6-\left(\mathrm{C}_{6} \mathrm{H}_{3}-2,6-\mathrm{Pr}_{2}\right)_{2}\right\}_{2}$ (3) (Nujol mull, CsI windows) 


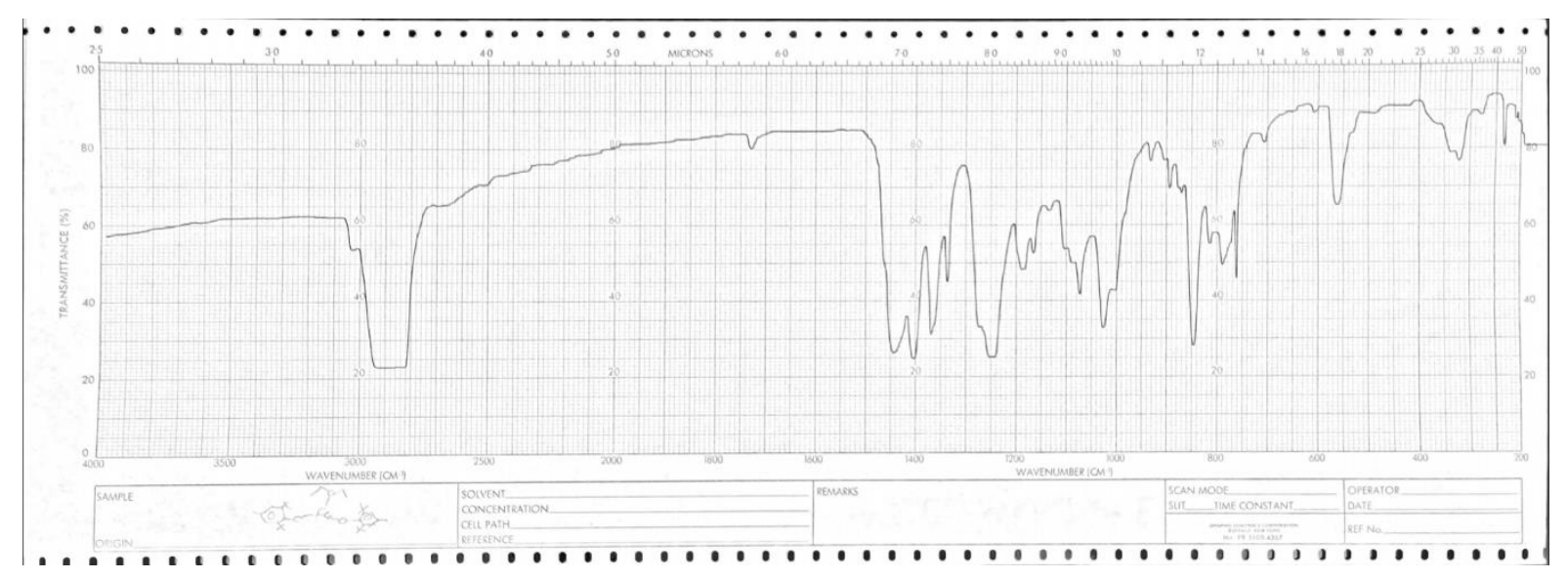

Figure S11: Infrared spectrum of $\mathrm{Fe}\left(\mathrm{OC}_{6} \mathrm{H}_{2}-2,6-\mathrm{Bu}_{2}^{\mathrm{t}}-4-\mathrm{Me}\right)_{2}\left(\mathrm{OEt}_{2}\right)(4)$ (Nujol mull, CsI windows)

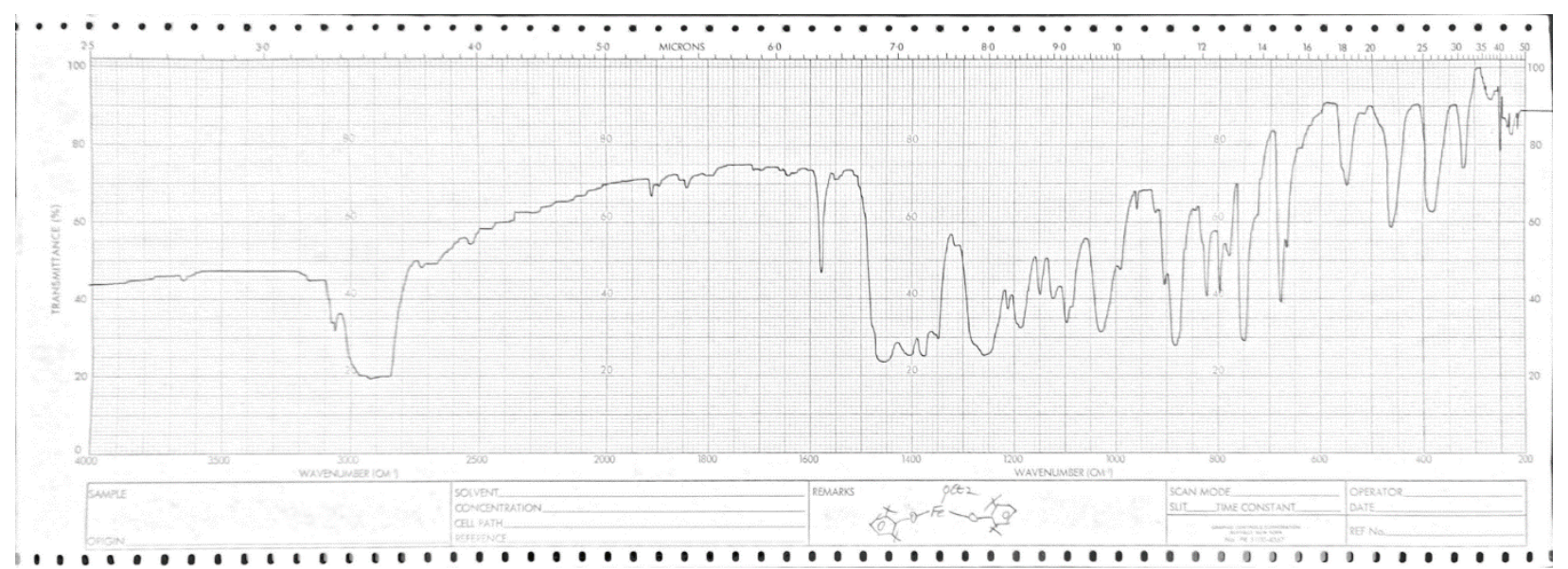

Figure S12: Infrared spectrum of $\mathrm{Fe}\left(\mathrm{OC}_{6} \mathrm{H}_{2}-2,6-\mathrm{Bu}_{2}\right)_{2}\left(\mathrm{OEt}_{2}\right)(5)$ (Nujol mull, Csl windows)

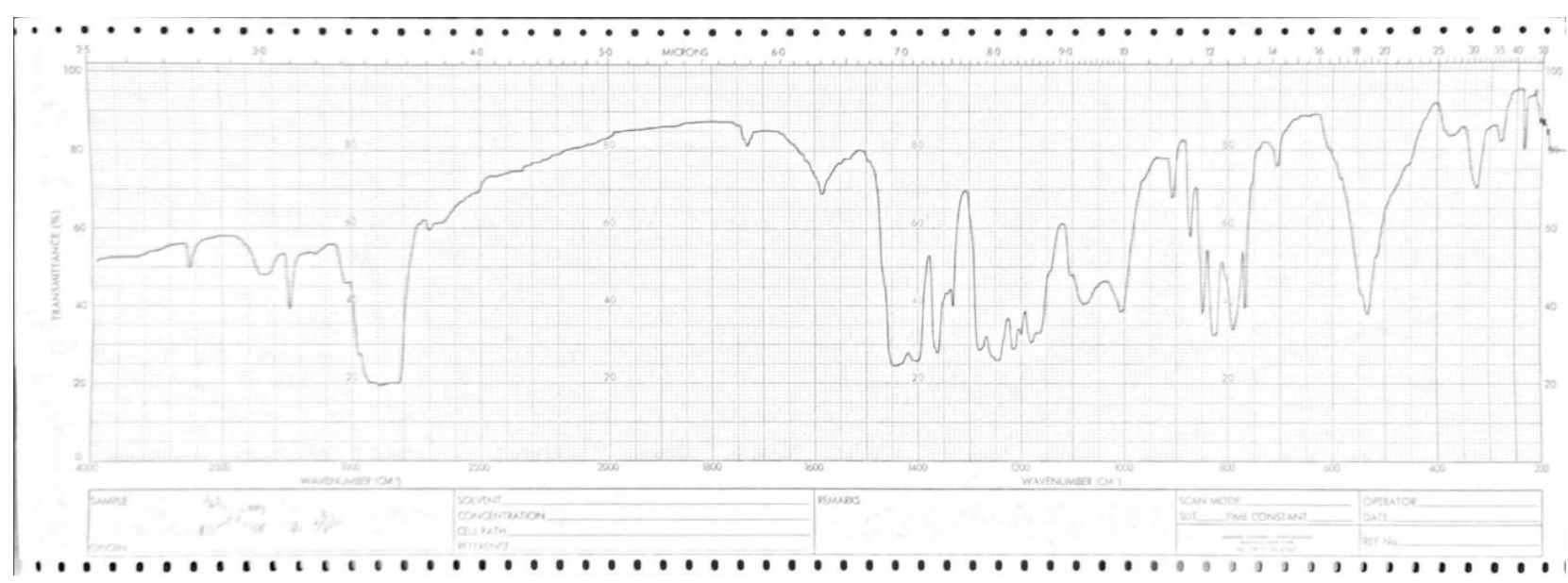

Figure S13: Infrared spectrum of $\mathrm{Fe}\left(\mathrm{OC}_{6} \mathrm{H}_{2}-2,6-\mathrm{Bu}_{2}{ }_{2}-4-\mathrm{Me}\right)_{2}\left(\mathrm{NH}_{3}\right)_{2}(6)$ (Nujol mull, CsI windows) 


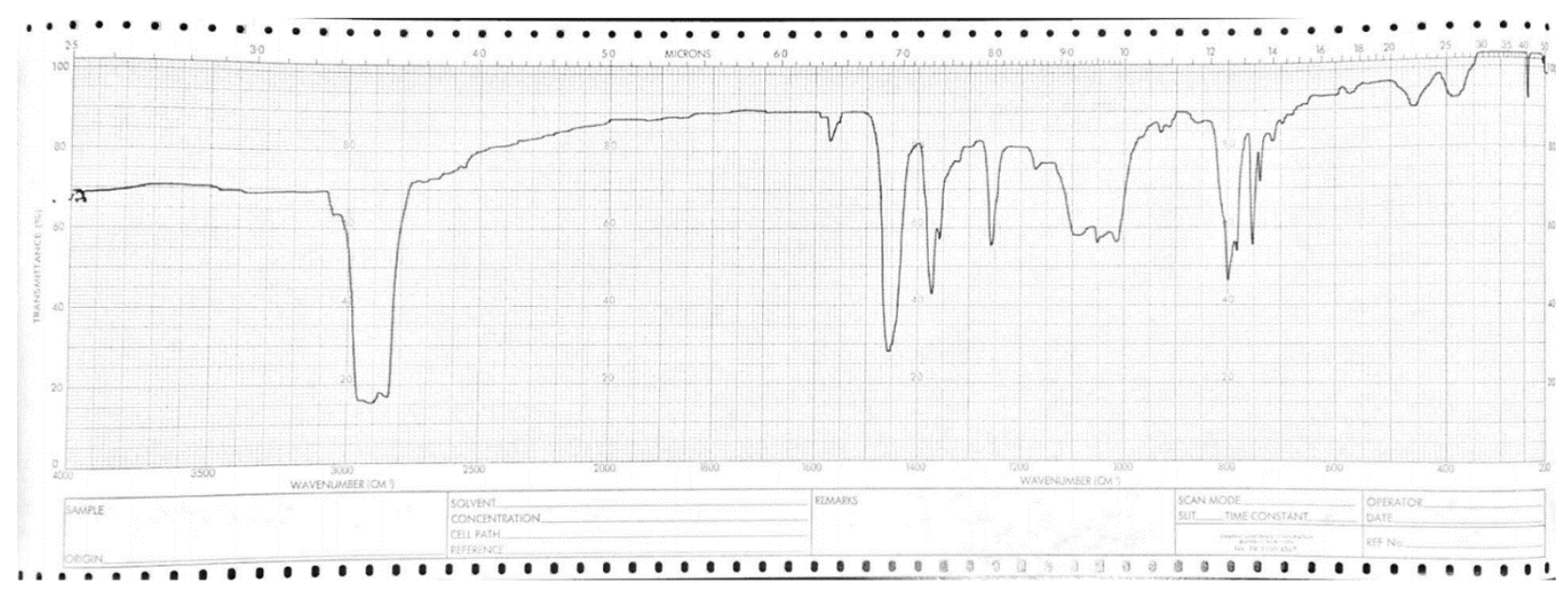

Figure S14: Infrared spectrum of $\mathrm{Fe}\left\{\mathrm{SC}_{6} \mathrm{H}_{3}-2,6-\left(\mathrm{C}_{6} \mathrm{H}_{3}-2,6-\mathrm{Pr}_{2}\right)_{2}\right\}_{2}\left(\mathrm{NH}_{3}\right)_{2}$ (7) (Nujol mull, CsI windows)

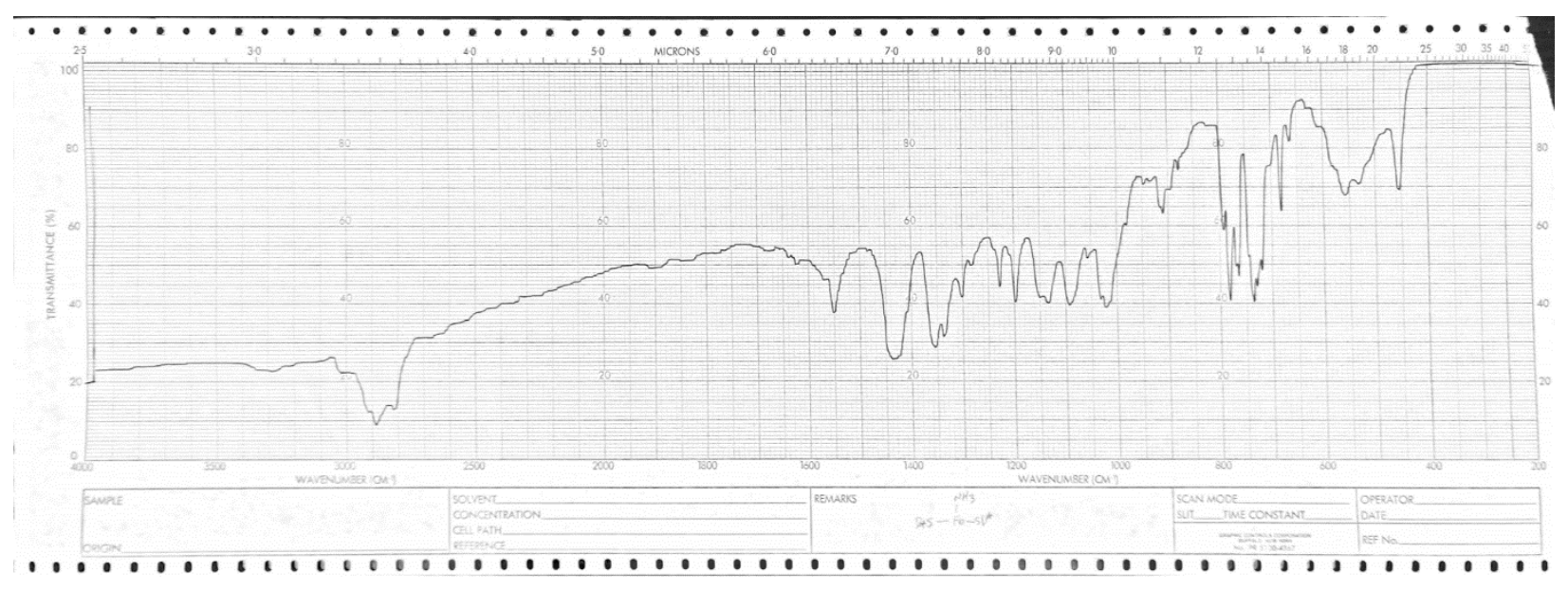

Figure S15: Infrared spectrum of $\mathrm{Fe}\left\{\mathrm{SC}_{6} \mathrm{H}_{3}-2,6-\left(\mathrm{C}_{6} \mathrm{H}_{3}-2,6-\mathrm{Pr}_{2}\right)_{2}\right\}_{2}\left(\mathrm{NH}_{3}\right)$ (8) (Nujol mull, CsI windows) 


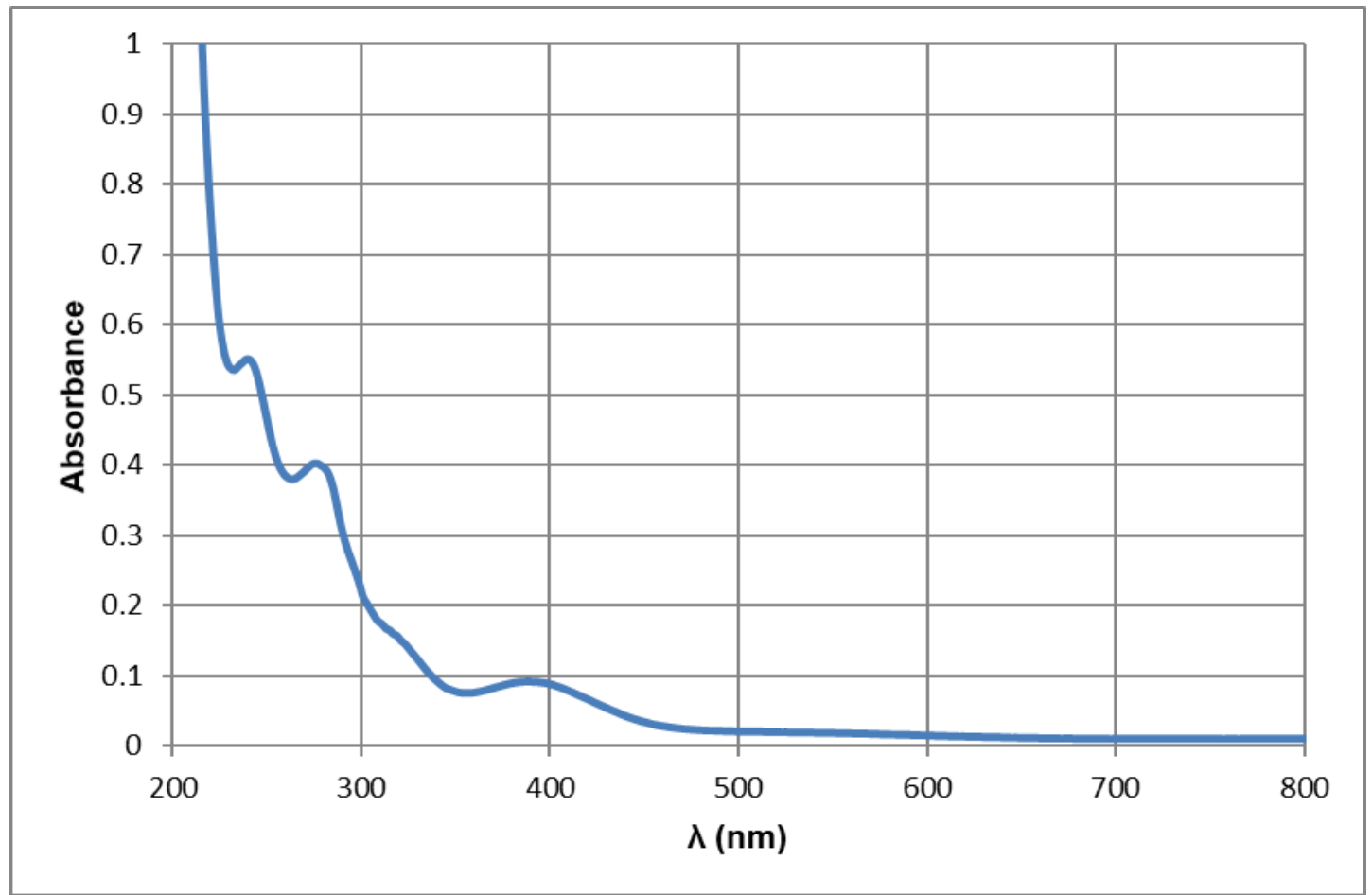

Figure S16: UV-Vis spectrum of $\left\{\mathrm{Fe}\left(\mathrm{OC}_{6} \mathrm{H}_{2}-2,6-\mathrm{Bu}_{2}{ }_{2}-4-\mathrm{Me}\right)_{2}\right\}_{2}(\mathbf{1})$ at $25^{\circ} \mathrm{C}(50 \mu \mathrm{M}$ in hexanes $)$

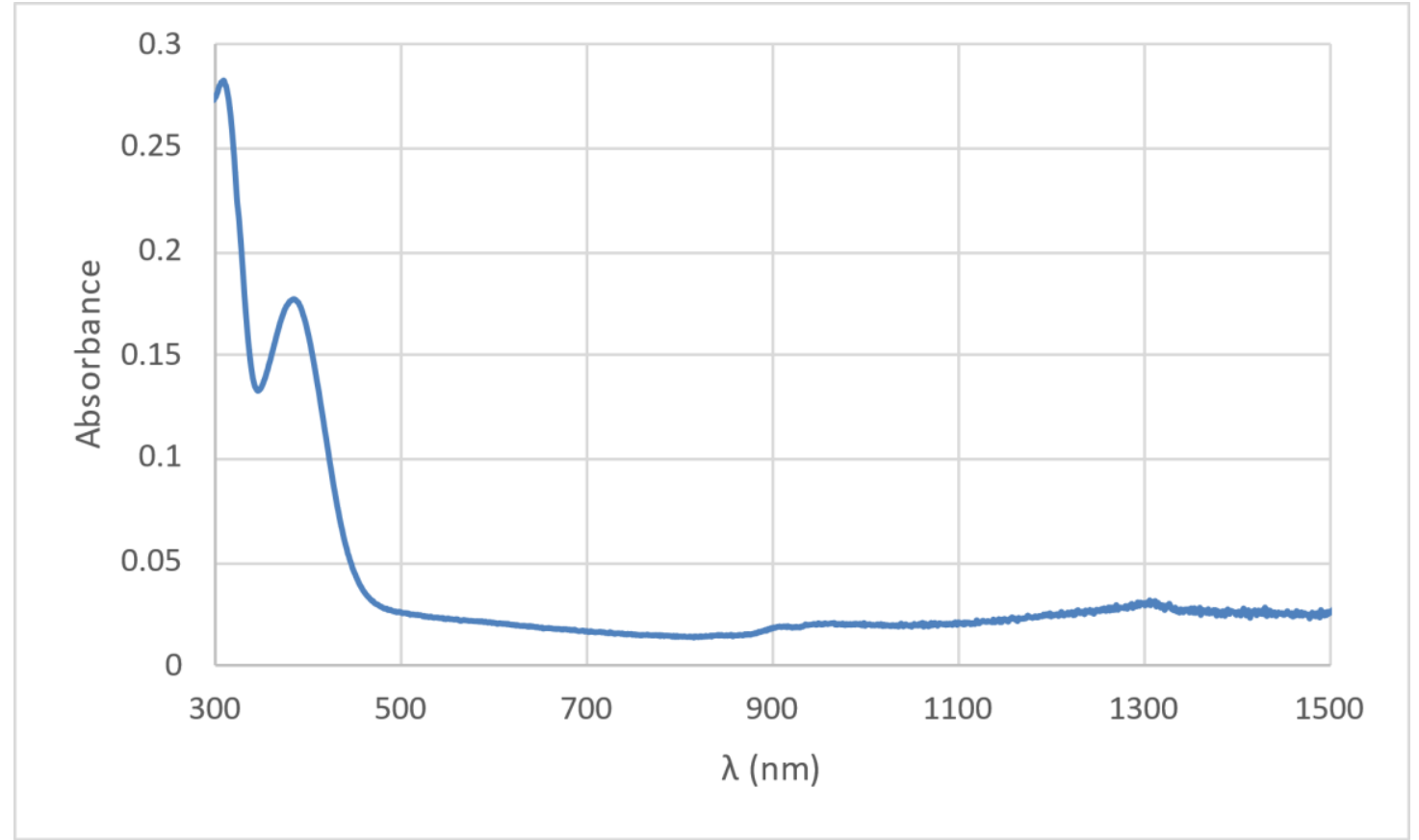

Figure S17: UV-Vis spectrum of $\left\{\mathrm{Fe}\left(\mathrm{OC}_{6} \mathrm{H}_{3}-2,6-\mathrm{Bu}_{2}^{\mathrm{t}}\right)_{2}\right\}_{2}(2)$ at $25^{\circ} \mathrm{C}(77 \mu \mathrm{M}$ in hexanes) 


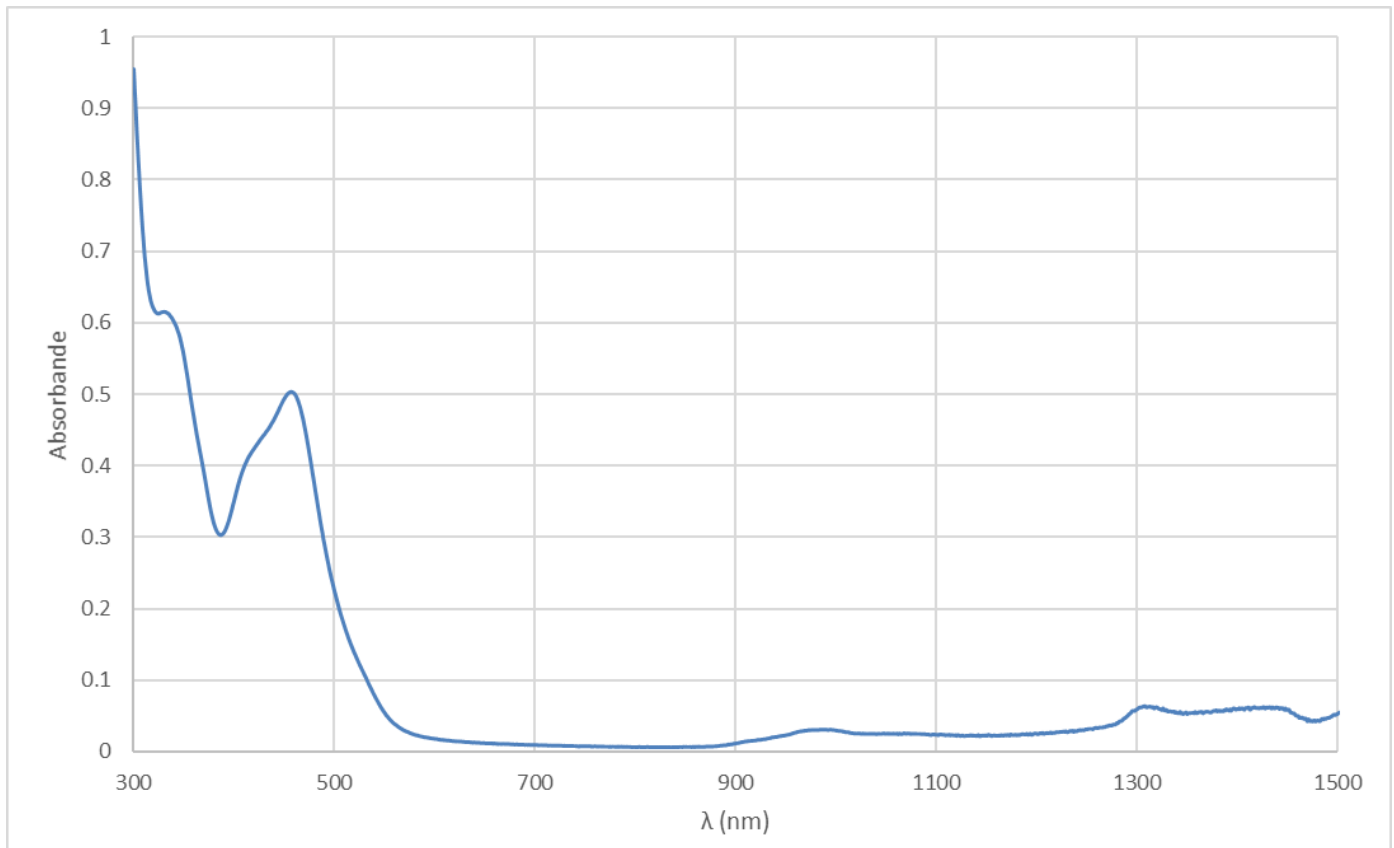

Figure S18: UV-Vis spectrum of Fe $\left\{\mathrm{SC}_{6} \mathrm{H}_{3}-2,6-\left(\mathrm{C}_{6} \mathrm{H}_{3}-2,6-\mathrm{Pr}_{2}\right)_{2}\right\}_{2}(3)$ at $25^{\circ} \mathrm{C}(160 \mu \mathrm{M}$ in hexanes)

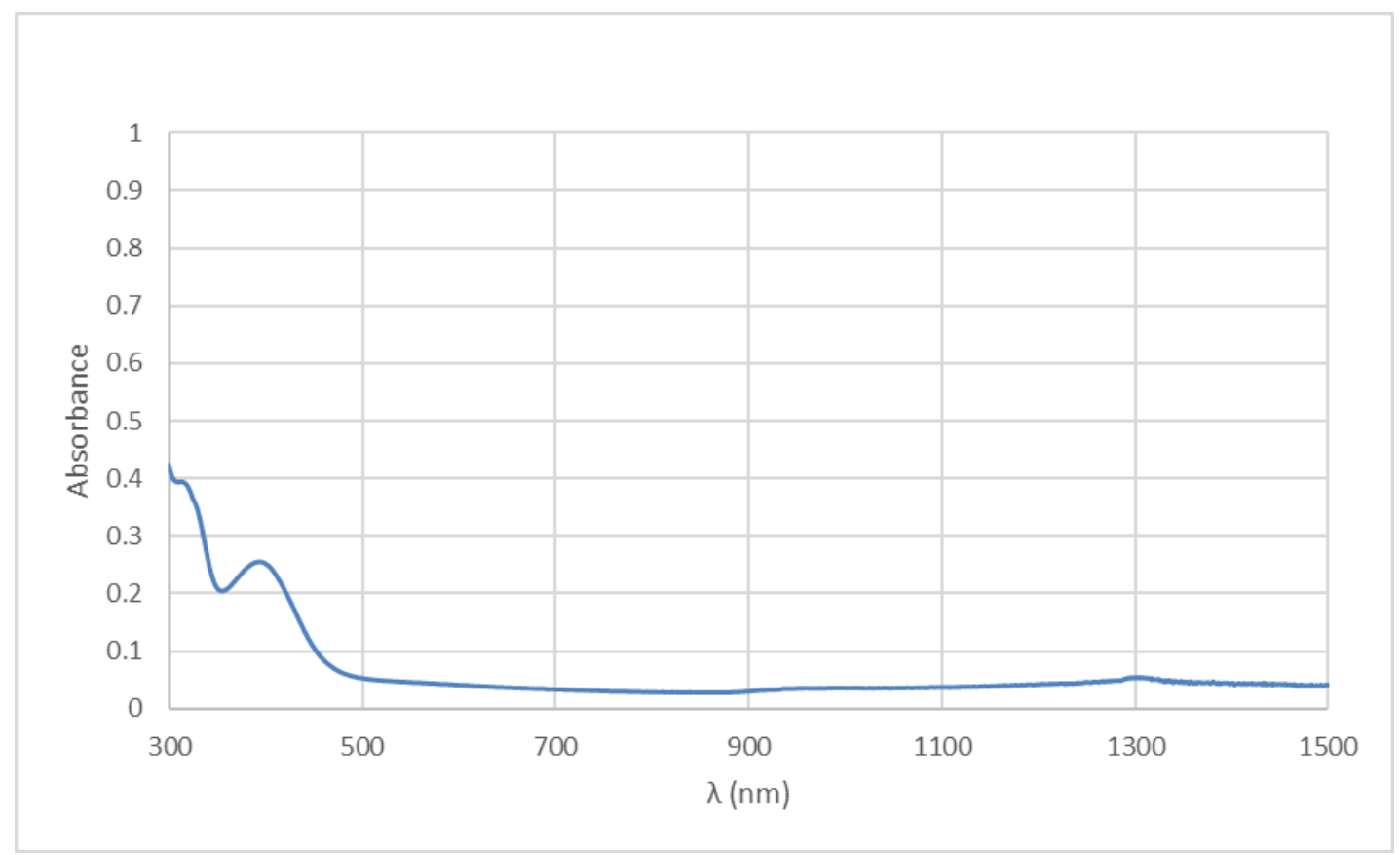

Figure S19: UV-Vis spectrum of $\mathrm{Fe}\left(\mathrm{OC}_{6} \mathrm{H}_{3}-2,6-\mathrm{Bu}_{2}{ }_{2}-4-\mathrm{Me}\right)_{2}\left(\mathrm{OEt}_{2}\right)(4)$ at $25{ }^{\circ} \mathrm{C}(130 \mu \mathrm{M}$ in hexanes) 


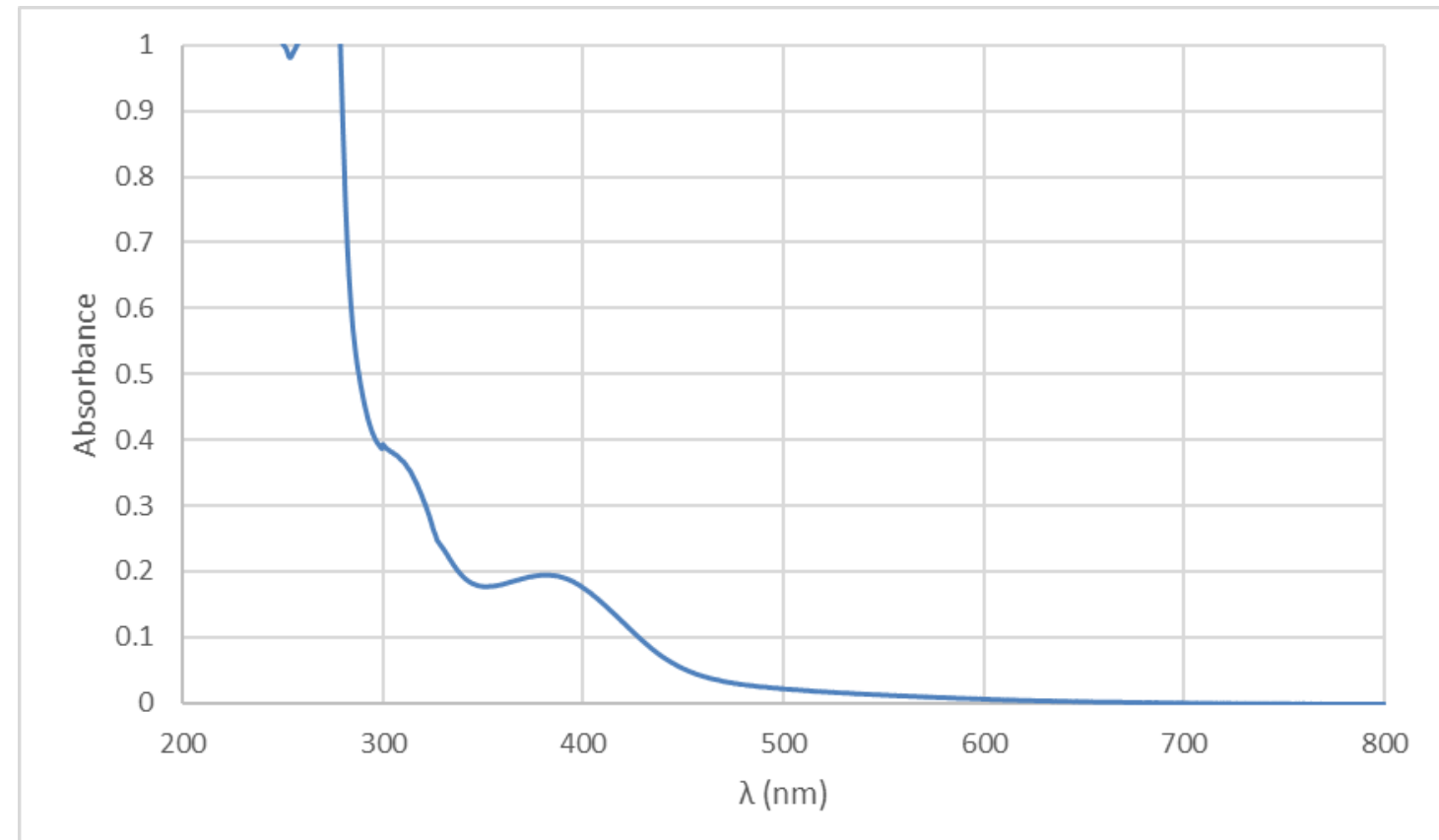

Figure S20: UV-Vis spectrum of $\mathrm{Fe}\left(\mathrm{OC}_{6} \mathrm{H}_{3}-2,6-\mathrm{Bu}_{2}\right)_{2}\left(\mathrm{OEt}_{2}\right)(5)$ at $25^{\circ} \mathrm{C}(280 \mu \mathrm{M}$ in hexanes)

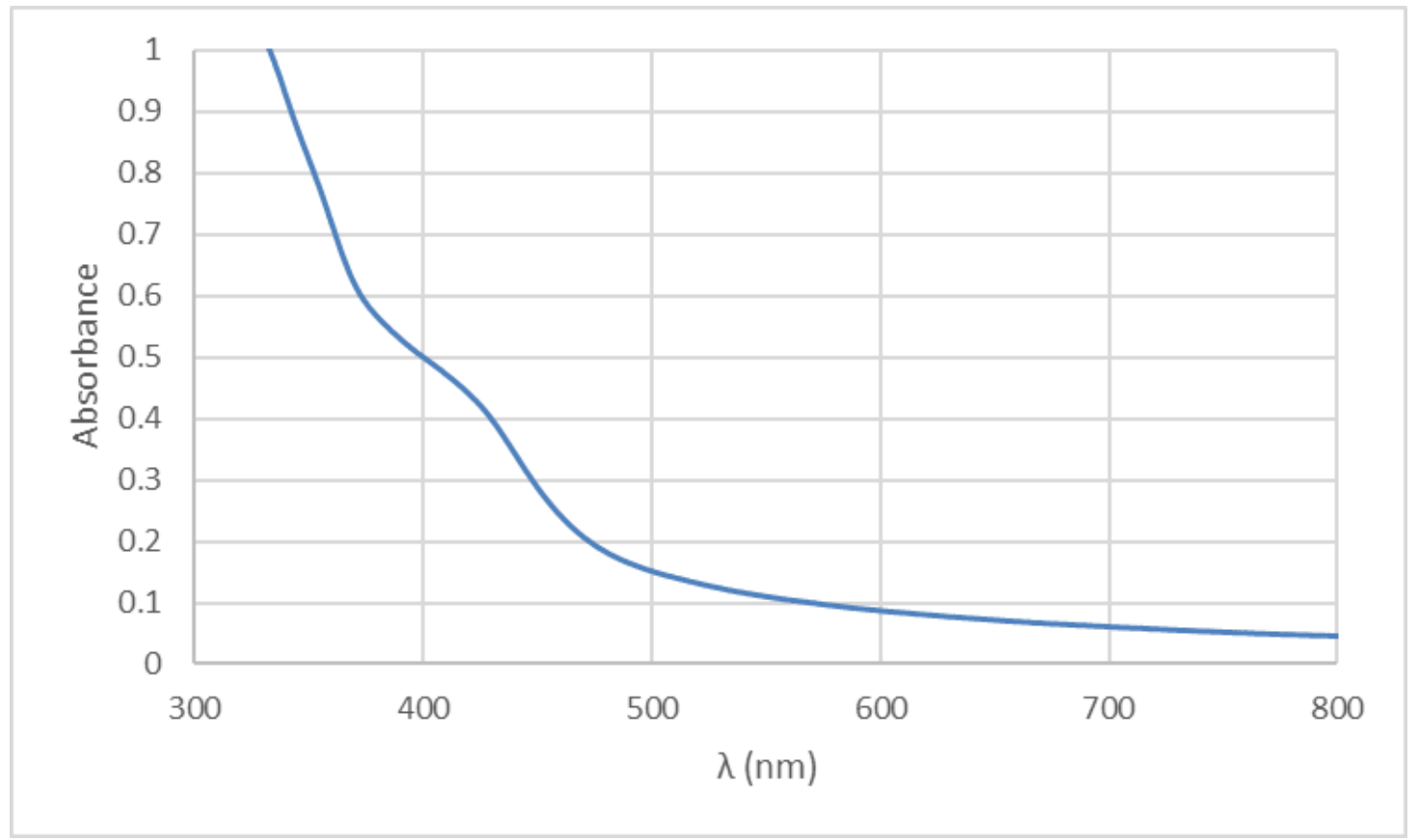

Figure S21: UV-Vis spectrum of $\mathrm{Fe}\left\{\mathrm{SC}_{6} \mathrm{H}_{3}-2,6-\left(\mathrm{C}_{6} \mathrm{H}_{3}-2,6-\mathrm{Pr}_{2}\right)_{2}\right\}_{2}\left(\mathrm{NH}_{3}\right)(8)$ at $25^{\circ} \mathrm{C}(1.1 \mathrm{mM}$ in hexanes) 
Table S1: Crystallographic and X-ray Data Collection Parameters of Complex 1

\begin{tabular}{|c|c|}
\hline Empirical formula & $\mathrm{C}_{66} \mathrm{H}_{105} \mathrm{Fe}_{2} \mathrm{O}_{4}$ \\
\hline Formula weight & 1074.19 \\
\hline Temperature/K & 90.15 \\
\hline Crystal system & triclinic \\
\hline Space group & $\mathrm{P}-1$ \\
\hline $\mathrm{a} / \AA$ & $13.8464(10)$ \\
\hline $\mathrm{b} / \AA$ & $14.4507(11)$ \\
\hline $\mathrm{c} / \AA$ & $16.8069(12)$ \\
\hline$\alpha /^{\circ}$ & $80.1450(11)$ \\
\hline$\beta /^{\circ}$ & $84.3778(10)$ \\
\hline$y /{ }^{\circ}$ & $70.2509(10)$ \\
\hline Volume $/ \AA^{3}$ & $3115.4(4)$ \\
\hline Z & 2 \\
\hline$\rho_{\text {calc }} \mathrm{g} / \mathrm{cm}^{3}$ & 1.145 \\
\hline$\mu / \mathrm{mm}^{-1}$ & 0.509 \\
\hline $\mathrm{F}(000)$ & 1170.0 \\
\hline Crystal size $/ \mathrm{mm}^{3}$ & $0.391 \times 0.357 \times 0.336$ \\
\hline Radiation & $\operatorname{MoKa}(\lambda=0.71073)$ \\
\hline $2 \Theta$ range for data collection $/{ }^{\circ}$ & 3.128 to 54.962 \\
\hline Index ranges & $-17 \leq \mathrm{h} \leq 17,-18 \leq \mathrm{k} \leq 18,-21 \leq \mathrm{I} \leq 21$ \\
\hline Reflections collected & 27884 \\
\hline Independent reflections & $14242\left[R_{\text {int }}=0.0156, R_{\text {sigma }}=0.0222\right]$ \\
\hline Data/restraints/parameters & $14242 / 33 / 731$ \\
\hline Goodness-of-fit on $\mathrm{F}^{2}$ & 1.044 \\
\hline Final $R$ indexes $[\mid>=2 \sigma(I)]$ & $R_{1}=0.0329, w R_{2}=0.0878$ \\
\hline Final $\mathrm{R}$ indexes [all data] & $R_{1}=0.0375, w R_{2}=0.09095$ \\
\hline Largest diff. peak/hole / e $\AA^{-3}$ & $0.47 /-0.40$ \\
\hline
\end{tabular}


Table S2: Crystallographic and X-ray Data Collection Parameters of Complex 2

\begin{tabular}{|c|c|}
\hline Empirical formula & $\mathrm{C}_{56} \mathrm{H}_{84} \mathrm{Fe}_{2} \mathrm{O}_{4}$ \\
\hline Formula weight & 932.93 \\
\hline Temperature/K & 90.15 \\
\hline Crystal system & monoclinic \\
\hline Space group & $\mathrm{C} 2 / \mathrm{c}$ \\
\hline $\mathrm{a} / \AA$ & $44.4824(19)$ \\
\hline $\mathrm{b} / \AA$ & $13.8567(6)$ \\
\hline $\mathrm{c} / \AA ̊ \AA$ & $19.7745(9)$ \\
\hline$\alpha /^{\circ}$ & 90 \\
\hline$\beta /^{\circ}$ & $113.916(2)$ \\
\hline $\mathrm{y} /{ }^{\circ}$ & 90 \\
\hline Volume $/ \AA^{3}$ & $11142.1(9)$ \\
\hline Z & 8 \\
\hline$\rho_{\text {calc }} \mathrm{g} / \mathrm{cm}^{3}$ & 1.112 \\
\hline$\mu / \mathrm{mm}^{-1}$ & 0.560 \\
\hline $\mathrm{F}(000)$ & 4032.0 \\
\hline Crystal size $/ \mathrm{mm}^{3}$ & $0.31 \times 0.225 \times 0.188$ \\
\hline Radiation & $\operatorname{MoKa}(\lambda=0.71073)$ \\
\hline $2 \Theta$ range for data collection $/^{\circ}$ & 4.068 to 59.442 \\
\hline Index ranges & $-61 \leq \mathrm{h} \leq 61,-19 \leq \mathrm{k} \leq 19,-27 \leq \mathrm{I} \leq 26$ \\
\hline Reflections collected & 123878 \\
\hline Independent reflections & $15792\left[R_{\text {int }}=0.0423, R_{\text {sigma }}=0.0267\right]$ \\
\hline Data/restraints/parameters & $15792 / 0 / 583$ \\
\hline Goodness-of-fit on $\mathrm{F}^{2}$ & 1.031 \\
\hline Final $R$ indexes $[\mid>=2 \sigma(I)]$ & $R_{1}=0.0504, w R_{2}=0.1419$ \\
\hline Final $\mathrm{R}$ indexes [all data] & $R_{1}=0.0710, w R_{2}=0.1566$ \\
\hline Largest diff. peak/hole / e $\AA^{-3}$ & $0.68 /-0.46$ \\
\hline
\end{tabular}


Table S3: Crystallographic and X-ray Data Collection Parameters of Complex 4

\begin{tabular}{|c|c|}
\hline Empirical formula & $\mathrm{C}_{34} \mathrm{H}_{56} \mathrm{FeO}_{3}$ \\
\hline Formula weight & 568.63 \\
\hline Temperature/K & 90.15 \\
\hline Crystal system & orthorhombic \\
\hline Space group & Pna21 \\
\hline $\mathrm{a} / \AA$ & 18.2039(5) \\
\hline $\mathrm{b} / \AA$ & 12.6572(3) \\
\hline $\mathrm{c} / \AA$ & 14.3079(4) \\
\hline$\alpha /^{\circ}$ & 90 \\
\hline$\beta /^{\circ}$ & 90 \\
\hline $\mathrm{y} /{ }^{\circ}$ & 90 \\
\hline Volume/ $/ \AA^{3}$ & $3296.69(15)$ \\
\hline Z & 4 \\
\hline$\rho_{\text {calc }} \mathrm{g} / \mathrm{cm}^{3}$ & 1.146 \\
\hline$\mu / \mathrm{mm}^{-1}$ & 0.487 \\
\hline $\mathrm{F}(000)$ & 1240.0 \\
\hline Crystal size $/ \mathrm{mm}^{3}$ & $0.371 \times 0.156 \times 0.11$ \\
\hline Radiation & $\operatorname{MoKa}(\lambda=0.71073)$ \\
\hline $2 \Theta$ range for data collection $/^{\circ}$ & 3.92 to 61.508 \\
\hline Index ranges & $-26 \leq \mathrm{h} \leq 26,-18 \leq \mathrm{k} \leq 18,-20 \leq \mathrm{I} \leq 20$ \\
\hline Reflections collected & 82435 \\
\hline Independent reflections & $10167\left[R_{\text {int }}=0.0668, R_{\text {sigma }}=0.0442\right]$ \\
\hline Data/restraints/parameters & $10167 / 1 / 359$ \\
\hline Goodness-of-fit on $\mathrm{F}^{2}$ & 1.061 \\
\hline Final $R$ indexes $[l>=2 \sigma(I)]$ & $R_{1}=0.0358, w R_{2}=0.0762$ \\
\hline Final $\mathrm{R}$ indexes [all data] & $R_{1}=0.0481, w R_{2}=0.0825$ \\
\hline Largest diff. peak/hole / e $\AA^{-3}$ & $0.44 /-0.28$ \\
\hline Flack parameter & $-0.002(6)$ \\
\hline
\end{tabular}


Table S4: Crystallographic and X-ray Data Collection Parameters of Complex 5

\begin{tabular}{|c|c|}
\hline Empirical formula & $\mathrm{C}_{32} \mathrm{H}_{52} \mathrm{FeO}_{3}$ \\
\hline Formula weight & 540.58 \\
\hline Temperature/K & 90.15 \\
\hline Crystal system & orthorhombic \\
\hline Space group & $P 2{ }_{12} 2$ \\
\hline $\mathrm{a} / \AA$ & $14.3983(16)$ \\
\hline $\mathrm{b} / \AA$ & $18.603(2)$ \\
\hline $\mathrm{c} / \AA$ & $11.6761(13)$ \\
\hline$\alpha /^{\circ}$ & 90 \\
\hline$\beta /^{\circ}$ & 90 \\
\hline$y /{ }^{\circ}$ & 90 \\
\hline Volume $/ \AA^{3}$ & $3127.4(6)$ \\
\hline Z & 4 \\
\hline$\rho_{\text {calc }} \mathrm{g} / \mathrm{cm}^{3}$ & 1.148 \\
\hline$\mu / \mathrm{mm}^{-1}$ & 0.510 \\
\hline $\mathrm{F}(000)$ & 1176.0 \\
\hline Crystal size $/ \mathrm{mm}^{3}$ & $1.264 \times 0.518 \times 0.493$ \\
\hline Radiation & $\operatorname{MoKa}(\lambda=0.71073)$ \\
\hline $2 \Theta$ range for data collection $/{ }^{\circ}$ & 3.488 to 65.026 \\
\hline Index ranges & $-21 \leq \mathrm{h} \leq 21,-27 \leq \mathrm{k} \leq 28,-17 \leq \mathrm{I} \leq 17$ \\
\hline Reflections collected & 51500 \\
\hline Independent reflections & $10806\left[R_{\text {int }}=0.0244, R_{\text {sigma }}=0.0273\right]$ \\
\hline Data/restraints/parameters & $10806 / 0 / 339$ \\
\hline Goodness-of-fit on $\mathrm{F}^{2}$ & 1.039 \\
\hline Final $R$ indexes $[l>=2 \sigma(I)]$ & $R_{1}=0.0251, w R_{2}=0.0645$ \\
\hline Final $\mathrm{R}$ indexes [all data] & $R_{1}=0.0278, w R_{2}=0.0658$ \\
\hline Largest diff. peak/hole / e $\AA^{-3}$ & $0.62 /-0.17$ \\
\hline Flack parameter & $0.008(2)$ \\
\hline
\end{tabular}


Table S5: Crystallographic and X-ray Data Collection Parameters of Complex 6

\begin{tabular}{|c|c|}
\hline Empirical formula & $\mathrm{C}_{98} \mathrm{H}_{176} \mathrm{Fe}_{3} \mathrm{~N}_{6} \mathrm{O}_{8}$ \\
\hline Formula weight & 1733.99 \\
\hline Temperature/K & 100.15 \\
\hline Crystal system & orthorhombic \\
\hline Space group & Pccn \\
\hline $\mathrm{a} / \AA$ & $32.1228(19)$ \\
\hline $\mathrm{b} / \AA$ & $12.9251(7)$ \\
\hline $\mathrm{c} / \AA ̊ \AA$ & $25.0112(12)$ \\
\hline$\alpha /^{\circ}$ & 90 \\
\hline$\beta /^{\circ}$ & 90 \\
\hline $\mathrm{y} /{ }^{\circ}$ & 90 \\
\hline Volume $/ \AA^{3}$ & $10384.4(10)$ \\
\hline Z & 4 \\
\hline$\rho_{\text {calc }} \mathrm{g} / \mathrm{cm}^{3}$ & 1.109 \\
\hline$\mu / \mathrm{mm}^{-1}$ & 0.465 \\
\hline $\mathrm{F}(000)$ & 3792.0 \\
\hline Crystal size $/ \mathrm{mm}^{3}$ & $0.483 \times 0.268 \times 0.106$ \\
\hline Radiation & $\operatorname{MoKa}(\lambda=0.71073)$ \\
\hline $2 \Theta$ range for data collection $/^{\circ}$ & 4.94 to 55 \\
\hline Index ranges & $-41 \leq \mathrm{h} \leq 41,-13 \leq \mathrm{k} \leq 16,-32 \leq \mathrm{I} \leq 32$ \\
\hline Reflections collected & 53778 \\
\hline Independent reflections & $11927\left[R_{\text {int }}=0.0392, R_{\text {sigma }}=0.0253\right]$ \\
\hline Data/restraints/parameters & $11927 / 30 / 588$ \\
\hline Goodness-of-fit on $\mathrm{F}^{2}$ & 1.037 \\
\hline Final $R$ indexes $[\mid>=2 \sigma(I)]$ & $R_{1}=0.0384, w R_{2}=0.0916$ \\
\hline Final $\mathrm{R}$ indexes [all data] & $R_{1}=0.0499, w R_{2}=0.0979$ \\
\hline Largest diff. peak/hole / e $\AA^{-3}$ & $0.29 /-0.32$ \\
\hline
\end{tabular}


Table S6: Crystallographic and X-ray Data Collection Parameters of Complex 7

\begin{tabular}{|c|c|}
\hline Empirical formula & $\mathrm{C}_{60} \mathrm{H}_{80} \mathrm{FeN}_{2} \mathrm{~S}_{2}$ \\
\hline Formula weight & 949.23 \\
\hline Temperature/K & 100.15 \\
\hline Crystal system & monoclinic \\
\hline Space group & $\mathrm{P} 2{ }_{1} / \mathrm{c}$ \\
\hline $\mathrm{a} / \AA$ & $10.8276(12)$ \\
\hline $\mathrm{b} / \AA$ & $19.563(2)$ \\
\hline $\mathrm{c} / \AA ̊ \AA$ & $26.586(3)$ \\
\hline$\alpha /^{\circ}$ & 90 \\
\hline$\beta /^{\circ}$ & $91.135(4)$ \\
\hline $\mathrm{y} /{ }^{\circ}$ & 90 \\
\hline Volume $/ \AA^{3}$ & $5630.4(11)$ \\
\hline Z & 4 \\
\hline$\rho_{\text {calc }} \mathrm{g} / \mathrm{cm}^{3}$ & 1.120 \\
\hline$\mu / \mathrm{mm}^{-1}$ & 0.379 \\
\hline $\mathrm{F}(000)$ & 2048.0 \\
\hline Crystal size $/ \mathrm{mm}^{3}$ & $0.434 \times 0.229 \times 0.227$ \\
\hline Radiation & $\operatorname{MoKa}(\lambda=0.71073)$ \\
\hline $2 \Theta$ range for data collection $/^{\circ}$ & 4.438 to 61.122 \\
\hline Index ranges & $-15 \leq \mathrm{h} \leq 15,-27 \leq \mathrm{k} \leq 27,-38 \leq \mathrm{I} \leq 37$ \\
\hline Reflections collected & 112722 \\
\hline Independent reflections & $17198\left[R_{\text {int }}=0.0326, R_{\text {sigma }}=0.0242\right]$ \\
\hline Data/restraints/parameters & $17198 / 0 / 604$ \\
\hline Goodness-of-fit on $\mathrm{F}^{2}$ & 1.031 \\
\hline Final $R$ indexes $[\mid>=2 \sigma(I)]$ & $R_{1}=0.0368, w R_{2}=0.0933$ \\
\hline Final $\mathrm{R}$ indexes [all data] & $R_{1}=0.0478, w R_{2}=0.0995$ \\
\hline Largest diff. peak/hole / e $\AA^{-3}$ & $0.51 /-0.43$ \\
\hline
\end{tabular}


Table S7: Crystallographic and X-ray Data Collection Parameters of Complex 8

\begin{tabular}{|c|c|}
\hline Empirical formula & $\mathrm{C}_{60} \mathrm{H}_{77} \mathrm{FeNS}_{2}$ \\
\hline Formula weight & 932.19 \\
\hline Temperature/K & 90.15 \\
\hline Crystal system & monoclinic \\
\hline Space group & $\mathrm{P} 2{ }_{1} / \mathrm{n}$ \\
\hline $\mathrm{a} / \AA$ & $13.452(3)$ \\
\hline $\mathrm{b} / \AA$ & $14.142(3)$ \\
\hline $\mathrm{c} / \AA ̊ \AA$ & $13.747(3)$ \\
\hline$\alpha /^{\circ}$ & 90 \\
\hline$\beta /^{\circ}$ & $94.616(3)$ \\
\hline $\mathrm{y} /{ }^{\circ}$ & 90 \\
\hline Volume $/ \AA^{3}$ & $2606.7(9)$ \\
\hline Z & 2 \\
\hline$\rho_{\text {calc }} \mathrm{g} / \mathrm{cm}^{3}$ & 1.188 \\
\hline$\mu / \mathrm{mm}^{-1}$ & 0.408 \\
\hline $\mathrm{F}(000)$ & 1004.0 \\
\hline Crystal size $/ \mathrm{mm}^{3}$ & $0.663 \times 0.519 \times 0.162$ \\
\hline Radiation & $\operatorname{MoKa}(\lambda=0.71073)$ \\
\hline $2 \Theta$ range for data collection $/^{\circ}$ & 4.076 to 61.238 \\
\hline Index ranges & $-19 \leq h \leq 19,-20 \leq k \leq 20,-19 \leq 1 \leq 19$ \\
\hline Reflections collected & 40807 \\
\hline Independent reflections & $8006\left[R_{\text {int }}=0.0366, R_{\text {sigma }}=0.0301\right]$ \\
\hline Data/restraints/parameters & $8006 / 0 / 316$ \\
\hline Goodness-of-fit on $\mathrm{F}^{2}$ & 1.052 \\
\hline Final $R$ indexes $[\mid>=2 \sigma(I)]$ & $R_{1}=0.0467, w R_{2}=0.1115$ \\
\hline Final $\mathrm{R}$ indexes [all data] & $R_{1}=0.0638, w R_{2}=0.1205$ \\
\hline Largest diff. peak/hole / e $\AA^{-3}$ & $0.43 /-0.36$ \\
\hline
\end{tabular}




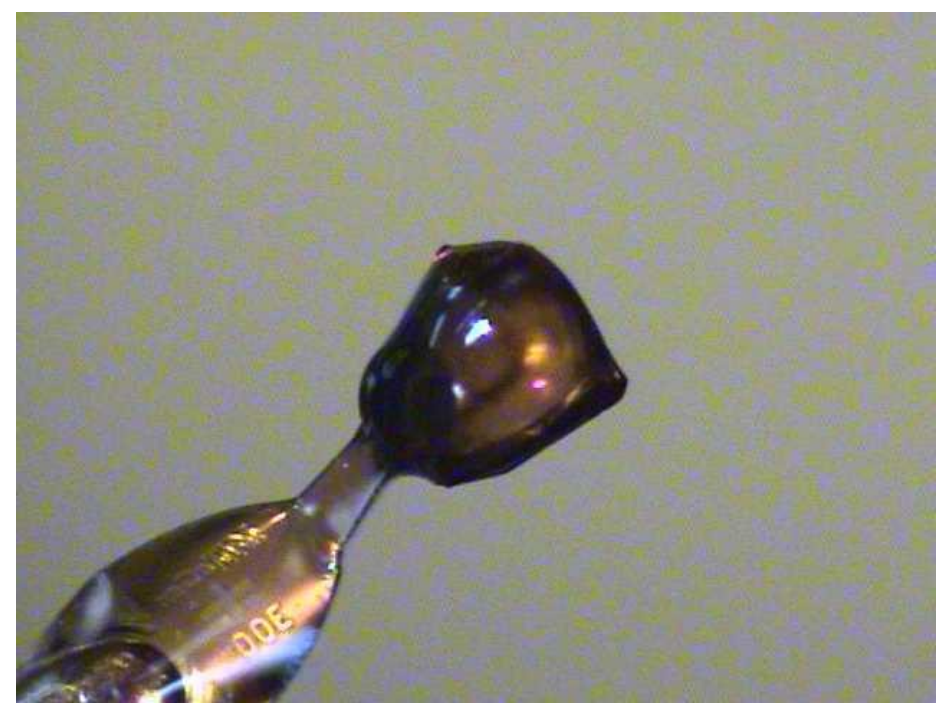

Figure S22: Photograph of crystalline $\left\{\mathrm{Fe}\left(\mathrm{OC}_{6} \mathrm{H}_{2}-2,6-\mathrm{Bu}^{t_{2}-}\right.\right.$ 4-Me) $\left.{ }_{2}\right\}_{2}(\mathbf{1})$

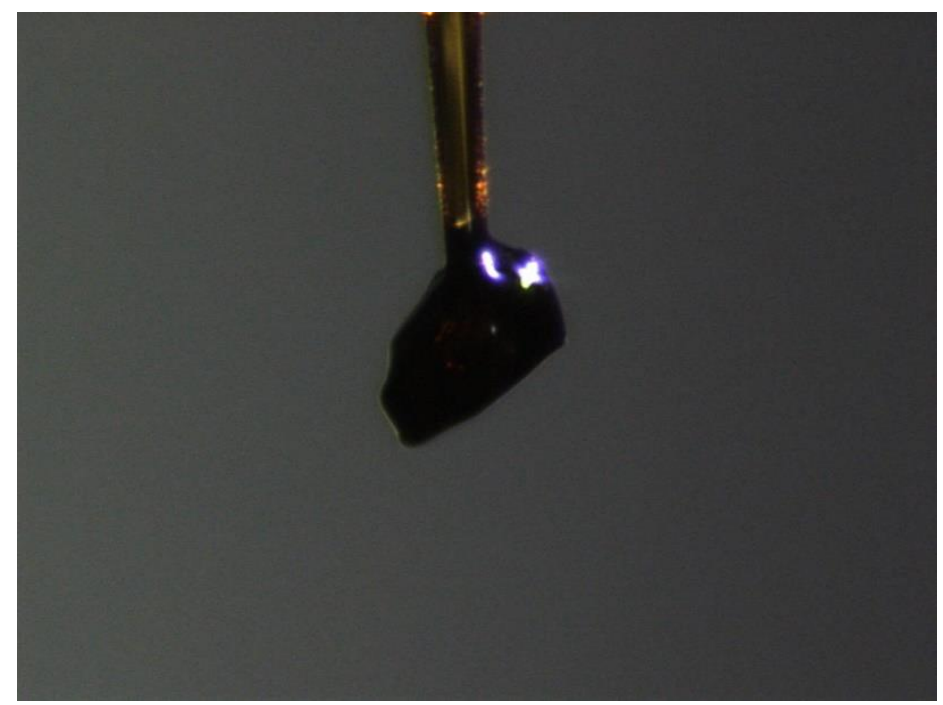

Figure S23: Photograph of crystalline $\left\{\mathrm{Fe}\left(\mathrm{OC}_{6} \mathrm{H}_{2}-2,6-\right.\right.$

$\left.\left.\mathrm{Bu}_{2}{ }_{2}\right)_{2}\right\}_{2}(2)$ 


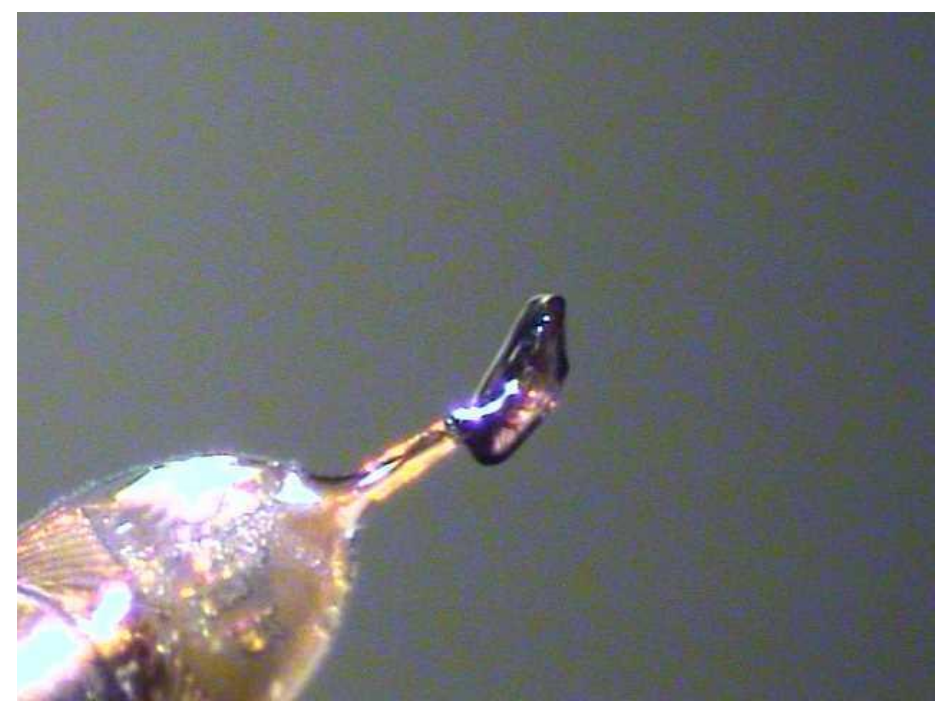

Figure S24: Photograph of crystalline $\left\{\mathrm{Fe}\left(\mathrm{OC}_{6} \mathrm{H}_{2}-2,6-\mathrm{Bu}^{\mathrm{t}} 2^{-}\right.\right.$ $\left.4-\mathrm{Me})_{2}\right\}_{2}\left(\mathrm{OEt}_{2}\right)(4)$

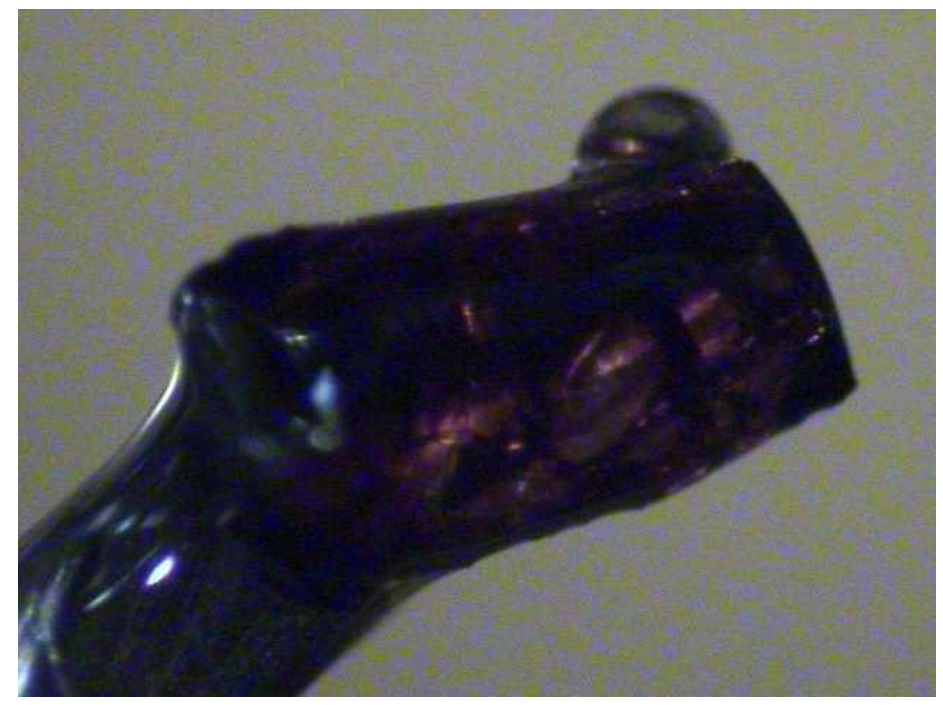

Figure S25: Photograph of crystalline $\left\{\mathrm{Fe}\left(\mathrm{OC}_{6} \mathrm{H}_{2}-2,6-\right.\right.$ $\left.\left.\mathrm{Bu}_{2}\right)_{2}\right\}_{2}\left(\mathrm{OEt}_{2}\right)(\mathbf{5})$ 


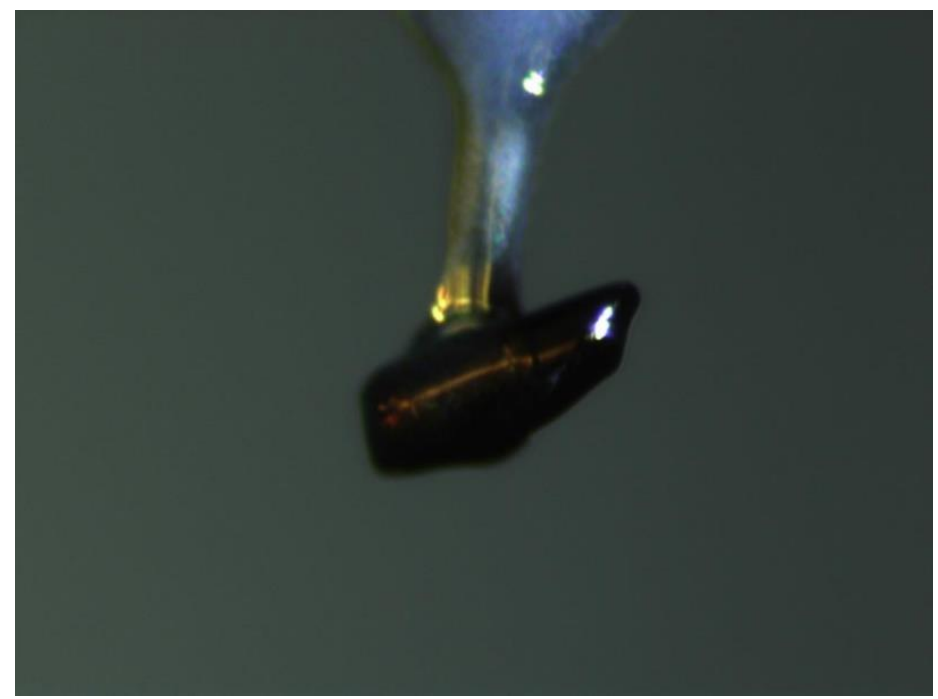

Figure S26: Photograph of crystalline $\left\{\mathrm{Fe}\left(\mathrm{OC}_{6} \mathrm{H}_{2}-2,6-\mathrm{Bu}_{2}{ }^{\mathrm{t}}-\right.\right.$ 4-Me) $\left.{ }_{2}\right\}_{2}\left(\mathrm{NH}_{3}\right)_{2}(\mathbf{6})$

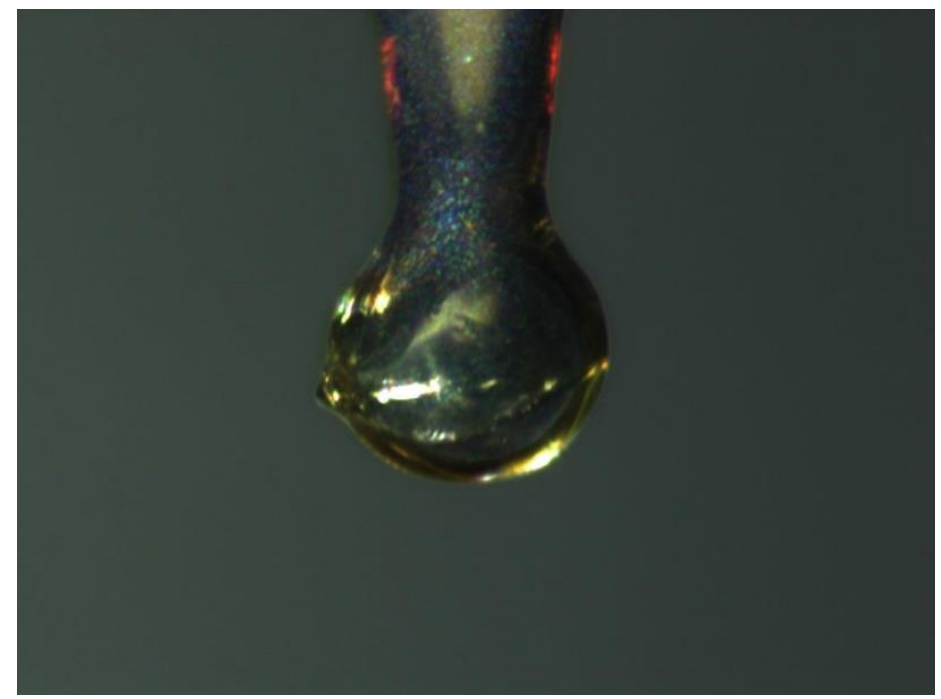

Figure S27: Photograph of crystalline $\mathrm{Fe}\left\{\mathrm{SC}_{6} \mathrm{H}_{3}-2,6-\left(\mathrm{C}_{6} \mathrm{H}_{3}-\right.\right.$ 2,6-Pri $\left.\left.{ }_{2}\right)_{2}\right\}_{2}\left(\mathrm{NH}_{3}\right)_{2}(7)$ 


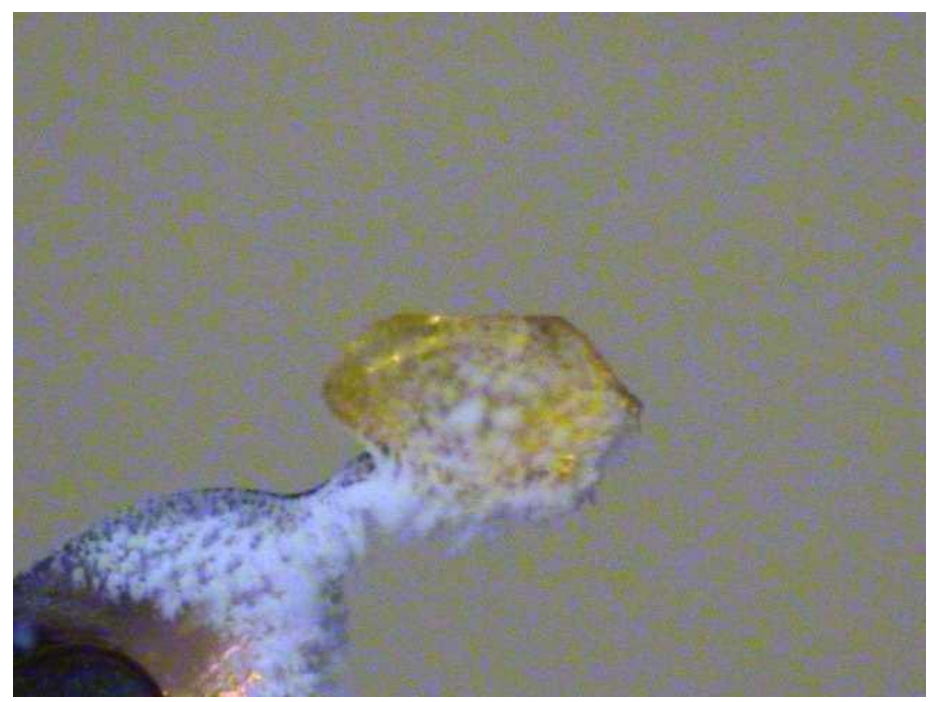

Figure S28: Photograph of crystalline $\mathrm{Fe}\left\{\mathrm{SC}_{6} \mathrm{H}_{3}-2,6-\left(\mathrm{C}_{6} \mathrm{H}_{3}-\right.\right.$ 2,6- $\left.\left.\operatorname{Pr}_{2}{ }_{2}\right)_{2}\right\}_{2}\left(\mathrm{NH}_{3}\right)(\mathbf{8})$ 